Boland, C. R. J., and B. O. Burwell. 2020. Ranking and mapping the high conservation priority bird species of Saudi Arabia. Avian Conservation and Ecology 15(2):18. https://doi.org/10.5751/ACE-01705-150218

Copyright (C) 2020 by the author(s). Published here under license by the Resilience Alliance.

Research Paper

\title{
Ranking and mapping the high conservation priority bird species of Saudi Arabia
}

\author{
Christopher R. J. Boland ${ }^{1}$ and Bruce O. Burwell ${ }^{2}$ \\ ${ }^{1}$ Environmental Protection Department, Saudi Aramco, ${ }^{2}$ Operational Excellence Department, Saudi Aramco
}

\begin{abstract}
One of the challenges facing conservation biologists and land managers is to determine which species in the biota should receive conservation priority. This is particularly challenging in regions with comparatively little baseline ecological data, such as the Arabian Peninsula. Here we create a list of high conservation priority species for the birds of Saudi Arabia using objective criteria. In total, 102 of Saudi Arabia's 401 regularly occurring bird species were assigned as high conservation priority. We then rank the conservation priority of these species based on their threatened status, population trend, national distribution and abundance, level of national responsibility, endemicity, and evolutionary distinctiveness. We use GIS to map the locations of high conservation priority species, revealing that most occur in the Kingdom's southwest (in the Asir Mountains and foothills, Tihama coastal plains, and Red Sea coastline). A wide array of threats is impacting Saudi Arabia's highest conservation priority birds, particularly overhunting, alpine habitat fragmentation and degradation, Allee effects, secondary poisoning, and persecution. The methodology that we developed could be easily applied to other taxa and in other countries to help identify and rank high conservation species in other regions with limited baseline data.
\end{abstract}

\section{Classement et cartographie des espèces d'oiseaux dont la conservation est hautement prioritaire en Arabie saoudite}

RÉSUMÉ. L'un des défis que doivent relever les biologistes de la conservation et les gestionnaires des terres consiste à déterminer quelles espèces du biote doivent être priorisées pour leur conservation. Cet exercice est particulièrement difficile dans les régions où les données écologiques de base sont relativement peu nombreuses, comme la péninsule arabique. Dans le présent article, nous avons établi une liste d'espèces prioritaires pour la conservation des oiseaux d'Arabie saoudite à partir de critères objectifs. En tout, 102 des 401 espèces d'oiseaux régulièrement présentes en Arabie Saoudite ont été classées comme hautement prioritaires pour leur conservation. Nous avons ensuite classé la priorité de ces espèces en fonction de leur statut de menace, de la tendance de leur population, de leur répartition et de leur abondance nationales, du niveau de responsabilité nationale, de leur endémicité et de leur caractère distinctif sur le plan de l'évolution. Nous avons utilisé le SIG pour cartographier leur présence, révélant que la plupart de ces espèces prioritaires se trouvent dans le sud-ouest du Royaume (dans les montagnes et les contreforts de l'Asir, les plaines côtières de Tihama et le littoral de la mer Rouge). Un large éventail de menaces pèse sur les oiseaux à priorité élevée de conservation en Arabie saoudite, notamment la chasse excessive, la fragmentation et la dégradation de l'habitat alpin, les effets de bordure, l'empoisonnement secondaire et la persécution. La méthodologie que nous avons élaborée pourrait facilement être appliquée à d'autres taxons et dans d'autres pays pour aider les responsables à identifier et à classer les espèces dont la conservation est prioritaire et où les données de base sont limitées.

Key Words: Asir Magpie; Asir Mountains; conservation priority; GIS; Saudi Arabia; threatened species

\section{INTRODUCTION}

Global biodiversity is experiencing an extinction rate a thousand times greater than the natural background rate (Pimm et al. 1995, Barnosky et al. 2011), and yet the resources currently made available for conservation are insufficient to prevent the impending loss of much of the world's threatened biodiversity (James et al. 2001). Therefore conservation agencies must prioritize which species receive active protection and which miss out, a concept known as conservation triage (Vane-Wright et al. 1991, Bottrill et al. 2008).

National conservation prioritization systems often vary greatly in what attributes are used, and how these attributes are scored and weighted (Schmeller et al. 2008, Le Berre et al. 2019). Most schemes score species based on extinction risk plus other measures of conservation importance, such as degree of endemicity, phylogenetic uniqueness, cost effectiveness, likelihood of success, and cultural, economic, or flagship value (e.g., Avery et al. 1995, Rodríguez et al. 2004, Gauthier et al. 2010, Seoane et al. 2011, Vieira da Silva et al. 2016). Ideally, these prioritization schemes are effective at drawing attention to high conservation priority species, helping conservation agencies make rational decisions, and ultimately conserving biodiversity.

Here we develop a simple and objective method for ranking the national conservation priority of vertebrate species using the birds of Saudi Arabia as a case study, a country with comparatively little baseline data, ecological research, or extant conservation programs (Meyer et al. 2015). There has been one previous 
attempt to prioritize Saudi Arabia's biodiversity. In 2003 the former National Commission for Wildlife Conservation and Development (NCWCD) produced a list of High Conservation Priority taxa (NCWCD 2003). However that list is now out of date and incomplete because of recent taxonomic revisions, revised global conservation assessments, new regional conservation assessments (Symes et al. 2015), substantially greater understanding of species distribution and abundance within Saudi Arabia (Jennings 2010, Boland and Alsuhaibany 2020), and a broader understanding of conservation prioritization principles in general (Wilson et al. 2006, 2009, Redding et al. 2008, Fischer et al. 2011, Jetz et al. 2014). Thus a new and updated list of high conservation priority species is warranted.

Our objectives are to (i) create an updated high conservation priority list of the birds of Saudi Arabia using objective criteria, (ii) develop a method to rank those species using quantifiable conservation attributes that could be repeated for other taxa or in other countries, and (iii) use GIS to identify locations of especially high conservation priority in Saudi Arabia. The aim of the updated list is to increase public awareness of the Kingdom's highest priority birds, encourage developers, industries, and private landowners to implement appropriate mitigation measures to avoid impacting species of highest conservation priority, and help inform the optimal allocation of limited conservation resources.

\section{METHODS}

\section{Ranking the high conservation priority species}

To compile the list of high conservation priority species we assessed all 401 bird species recorded with confidence in Saudi Arabia (Boland and Alsuhaibany 2020). Species were regarded as high conservation priority if they met one or more of the following three criteria. First, species were deemed high conservation priority if they are globally or regionally vulnerable, endangered, critically endangered, regionally extinct, or reintroduced (Symes et al. 2015, IUCN 2020). Second, species were deemed high conservation priority if they are regionally endemic (i.e., they occur entirely within the Arabian Peninsula south of Saudi Arabia's northern border, allowing for vagrant sightings) or near endemic (where more than $95 \%$ of their range occurs within the Arabian Peninsula), or contain regionally endemic subspecies (as per del Hoyo et al. 2020). Finally, we deemed species as high conservation priority if more than $50 \%$ of their global population occurs within Saudi Arabia at some stage during the year. For resident species, we conservatively estimated the proportion of the global population occurring in Saudi Arabia based on global distribution maps, assuming species have a homogenous distribution within their range. For migratory species, the percentage of the global population that is likely to pass through or winter in the Kingdom was estimated based upon the position of Saudi Arabia in relation to the breeding and wintering ranges, assuming species migrate in a direct route between their breeding and nonbreeding ranges (as depicted in del Hoyo et al. 2020).

To rank the high conservation priority species we applied a weighted scoring system based on measurable aspects of conservation significance as shown in Table 1. For each high conservation priority species, we scored the following attributes: global conservation status, regional conservation status, global population trend, regional population trend, percentage of global population occurring in Saudi Arabia, level of endemicity, national abundance, area of occurrence, and evolutionary distinctiveness. Our scoring approach for each attribute is outlined in turn below.

First, species with poorer conservation statuses were scored higher, as were species with decreasing populations and species with a greater percentage of the global population occurring in Saudi Arabia (Table 1). Species that are endemic to the Arabian Peninsula were scored higher than species that are near endemic, which in turn were scored higher than species that contain endemic subspecies. Populations that are not endemic or near endemic to the Arabian Peninsula were scored 0 (Table 1).

To score the abundance of breeding species within Saudi Arabia, the estimated number of breeding pairs (from Boland and Alsuhaibany 2020) was assigned a score out of 10 (see Table 1). Species with lower breeding abundances were scored higher. Former breeding species that are now possibly extinct within the Kingdom, namely, Arabian Bustard (Ardeotis arabs), Bateleur (Terathopius ecaudatus), and Bearded Vulture (Gypaetus barbatus), were treated as breeding species and given the maximum score. For nonbreeding birds, the relative abundance of each species (from Boland and Alsuhaibany 2020) was assigned a score out of 10 (see Table 1). Scarcer species were scored higher than more abundant species. Nonbreeding species that are both passage migrants and winter visitors had their abundance scores for these traits averaged. Species that have both a breeding and nonbreeding range within Saudi Arabia had their nonbreeding abundance scores excluded.

To score the area of occurrence within Saudi Arabia for each species, first we used ArcGIS 10.6.1 (ESRI 2018) to create GIS polygons from published breeding, wintering, and passage range maps (Boland and Alsuhaibany 2020). The percentage of Saudi Arabia's land area occupied by each species was then calculated using GIS and scored as per Table 1. Breeding ranges were scored out of 10 , whereas wintering and passage ranges were each scored out of 5 . Species that have both a breeding and nonbreeding range within Saudi Arabia had their nonbreeding range scores excluded. Species that are confined to a smaller area within Saudi Arabia scored higher.

Finally, evolutionary distinctiveness was scored out of 10 on three attributes of equal weight: (i) the number of species in the genera globally, (ii) the number of species in the family globally, and (iii) the number of species in the genera within Saudi Arabia (del Hoyo et al. 2020, Boland and Alsuhaibany 2020). Species that are more evolutionarily distinct scored higher (Table 1).

The scoring system was applied with the following caveats. Regional conservation status and trend had not been reported for 12 of the species included in the final list (see Table 2); therefore we scored the regional status as per its global status and the regional trend as "unknown." The regional population trend for Arabian Lark (Eremalauda eremodites) was scored as decreasing because of the dearth of recent sightings (Boland and Alsuhaibany 2020) although it is listed as stable by the IUCN 
(Symes et al. 2015). The Arabian Spotted Eagle-Owl (Bubo africanus milesi) is treated as a "probable regional endemic" (Boland and Alsuhaibany 2020, Holt et al. 2020), and was assigned a score of 7.5 , whereas a recognized endemic species would score 10. Finally, we assigned the Steppe Whimbrel (Numenius phaeopus alboaxillaris) as critically endangered regionally with an unknown population trend. Because the Steppe Whimbrel is a subspecies, it was assigned a score of 5 for its critical status, whereas a critically endangered full species would score 10.

Table 1. The scoring system used to rank high conservation priority species. Figures in parentheses show the maximum score for each attribute.

\begin{tabular}{|c|c|c|}
\hline Attribute & Classification & Score \\
\hline \multicolumn{3}{|l|}{ Global attributes [20] } \\
\hline \multirow[t]{5}{*}{ Global conservation status } & $\begin{array}{l}\text { Critically } \\
\text { endangered }\end{array}$ & 10 \\
\hline & Endangered & 7.5 \\
\hline & Vulnerable & 5 \\
\hline & Near threatened & 2.5 \\
\hline & Least concern & 0 \\
\hline \multirow[t]{3}{*}{ Global population trend } & Decreasing & 10 \\
\hline & Stable or unknown & 5 \\
\hline & Increasing & 0 \\
\hline \multicolumn{3}{|l|}{ Regional attributes [20] } \\
\hline \multirow[t]{6}{*}{ Regional conservation status } & Reintroduced & 10 \\
\hline & $\begin{array}{l}\text { Critically } \\
\text { endangered }\end{array}$ & 10 \\
\hline & Endangered & 7.5 \\
\hline & Vulnerable & 5 \\
\hline & Near threatened & 2.5 \\
\hline & Least concern & 0 \\
\hline \multirow[t]{5}{*}{ Regional population trend } & Decreasing & 10 \\
\hline & Possibly decreasing & 7.5 \\
\hline & Stable or unknown & 5 \\
\hline & Possibly increasing & 2.5 \\
\hline & Increasing & 0 \\
\hline \multicolumn{3}{|l|}{ National attributes [40] } \\
\hline \multirow[t]{11}{*}{ Breeding pairs } & Possibly extinct & 10 \\
\hline & $1-9$ & 9 \\
\hline & $10-99$ & 8 \\
\hline & $100-499$ & 7 \\
\hline & $500-999$ & 6 \\
\hline & $1000-4999$ & 5 \\
\hline & $5000-9999$ & 4 \\
\hline & $10,000-19,999$ & 3 \\
\hline & $20,000-99,999$ & 2 \\
\hline & $100,000-299,999$ & 1 \\
\hline & 300,000 or more & 0 \\
\hline \multirow[t]{7}{*}{ Nonbreeding abundance } & Possibly extinct & 10 \\
\hline & Extremely rare & 8 \\
\hline & Rare & 6 \\
\hline & Scarce & 4 \\
\hline & Uncommon & 2 \\
\hline & Common or & 0 \\
\hline & Abundant & \\
\hline Breeding or nonbreeding area $[\%$ of Saudi & 0.5 or less & 10 \\
\hline \multirow[t]{7}{*}{ Arabia] } & 1 & 9 \\
\hline & 2 & 8 \\
\hline & etc. & $\ldots$ \\
\hline & 7 & 3 \\
\hline & 8 & 2 \\
\hline & 9 & 1 \\
\hline & 10 or more & 0 \\
\hline
\end{tabular}

(con'd)

\begin{tabular}{llc} 
\% of global population in Saudi Arabia & 100 & 20 \\
& 90 & 18 \\
& 80 & 16 \\
& etc. & $\ldots$ \\
& 10 & 2 \\
Level of endemicity [10] & 1 & 0.2 \\
Endemic species status & Regional endemic & 10 \\
& Near endemic & 7.5 \\
& Probable endemic & 7.5 \\
Endemic subspecies status & Regional endemic & 5 \\
& Near endemic & 2.5 \\
Evolutionary distinctiveness [10] & 1 & \\
Number of species in the genus & $2-5$ & 3.3 \\
& $6-10$ & 2.5 \\
& $11-20$ & 1.7 \\
& 21 or more & 0.8 \\
Number of species in the family & 1 & 0 \\
& $2-5$ & 3.3 \\
& $6-10$ & 2.5 \\
& $11-20$ & 1.7 \\
Number of species in the genus in Saudi & 21 or more & 0.8 \\
Arabia & 1 & 0 \\
& $2-5$ & 3.3 \\
& $6-10$ & 2.5 \\
& $11-20$ & 1.7 \\
& 21 or more & 0.8 \\
& Maximum & 0 \\
& & 100 \\
\hline
\end{tabular}

\section{Mapping the high conservation priority species}

We mapped the summed conservation priority scores for all 102 high conservation priority species using ArcGIS 10.6.1 (ESRI 2018). The range maps of all high conservation priority species were clipped according to each species' elevation limits (as reported in Boland and Alsuhaibany 2020). Each range layer was converted to a raster format using the conservation priority score as its attribute value. Each species' individual raster layers were combined using weighted sum where each layer was given a weighting factor of 1 . In other words, the range maps for each species were overlain, and the sum of conservation priority scores for each species at each point in the Kingdom was mapped. We used the SETNULL function to identify which points contained either the maximum or the minimum summed conservation priority scores for all species. We then used the Locate Region Tool to identify which $100-\mathrm{km}^{2}$ patches contained either the maximum or the minimum average summed conservation priority scores.

\section{RESULTS}

\section{Ranking the high conservation priority species}

A total of 102 species made the list of high conservation priority taxa (Table 2). Seventeen species were included because of their global threatened conservation status, while 29 species were included because they have been assessed as regionally threatened (Symes et al. 2015), including 10 species that were already on the list on account of their international conservation status. Twenty species met the criteria for inclusion on the list because of their regional endemic status: 15 species are fully 
Table 2. Saudi Arabia's high conservation priority bird species.

Key: ${ }^{\dagger}$ included on NCWCD List; ${ }^{\ddagger}$ nonbreeding species; ${ }^{\$}$ regional status and trend had not been assessed; ${ }^{9}$ species-specific caveat (see methods).

Status: score $[\mathrm{CR}=$ critically endangered; $\mathrm{EN}=$ endangered; $\mathrm{VU}=$ vulnerable; $\mathrm{NT}=$ near threatened; $\mathrm{LC}=$ least concern]; Trend: score $[\mathrm{DE}=$ decreasing; $\mathrm{PD}=$ possibly decreasing; $\mathrm{ST}=$ stable; $\mathrm{PI}=$ possibly increasing; $\mathrm{IN}=$ increasing; $\mathrm{UN}=$ unknown]; Area = area of occurrence within Saudi Arabia: score [\% of Saudi Arabia]; Abundance: score [estimated number of breeding pairs for breeding species or relative abundance for nonbreeding species]; Global population: score [percentage of global population that occurs within Saudi Arabia during the year]; Endemicity: score [EA = Endemic to Arabia; NE = near endemic to Arabia; PE = probably endemic to Arabia; WS = widespread species (not endemic); ES = endemic subspecies; NS = near endemic subspecies; Distinctness: score [number of species in the genera - number of species in the family - number of species in the genera within Saudi Arabia].

\begin{tabular}{|c|c|c|c|c|c|c|c|c|c|c|c|}
\hline Rank & Species & Score & $\begin{array}{l}\text { Global } \\
\text { Status }\end{array}$ & $\begin{array}{l}\text { Global } \\
\text { Trend }\end{array}$ & $\begin{array}{l}\text { Regional } \\
\text { Status }\end{array}$ & $\begin{array}{l}\text { Regional } \\
\text { Trend }\end{array}$ & Area & Abundance & $\begin{array}{c}\text { Global } \\
\text { Population }\end{array}$ & Endemicity & Distinctness \\
\hline 1 & $\begin{array}{l}\text { Asir Magpie }{ }^{\dagger} \\
\text { Pica asirensis }\end{array}$ & 87.8 & $7.5[\mathrm{EN}]$ & $10[\mathrm{DE}]$ & $7.5[\mathrm{EN}]$ & $10[\mathrm{DE}]$ & $10[0.2]$ & $7[130]$ & 20 [100] & $10[\mathrm{EA}]$ & $5.8[5-130-1]$ \\
\hline 2 & $\begin{array}{l}\text { Basra Reed-Warbler } \\
\text { Acrocephalus griseldis }\end{array}$ & 62.0 & $7.5[\mathrm{EN}]$ & $5[\mathrm{ST}]$ & $7.5[\mathrm{EN}]$ & $5[\mathrm{UN}]$ & $10[0.2]$ & $9[8]$ & $18[90]$ & 0 [WS] & 0 [37-54-7] \\
\hline 3 & $\begin{array}{l}\text { Socotra Cormorant } \\
\text { Phalacrocorax nigrogularis }\end{array}$ & 61.6 & $5[\mathrm{VU}]$ & $10[\mathrm{DE}]$ & 5 [VU] & $10[\mathrm{DE}]$ & $9[1]$ & $2[35,000]$ & 6.4 [32] & $10[\mathrm{EA}]$ & $4.2[11-35-1]$ \\
\hline 4 & $\begin{array}{l}\text { Arabian Woodpecker } \\
\text { Dendropicos dorae }\end{array}$ & 60.8 & $2.5[\mathrm{NT}]$ & $10[\mathrm{DE}]$ & $2.5[\mathrm{NT}]$ & $10[\mathrm{DE}]$ & 3 [7] & $4[5500]$ & $14.6[73]$ & $10[\mathrm{EA}]$ & $4.2[17-254-1]$ \\
\hline 5 & $\begin{array}{l}\text { Yemen Warbler } \\
\text { Sylvia buryi }\end{array}$ & 60.2 & $2.5[\mathrm{NT}]$ & $10[\mathrm{DE}]$ & $2.5[\mathrm{NT}]$ & $10[\mathrm{DE}]$ & $10[0.1]$ & $4[5000]$ & $11.2[56]$ & $10[\mathrm{EA}]$ & 0 [30-69-13] \\
\hline 6 & $\begin{array}{l}\text { Asian Houbara } \\
\text { Chlamydotis macqueenii }^{\dagger}\end{array}$ & 59.8 & $5[\mathrm{VU}]$ & $10[\mathrm{DE}]$ & 10 [CR] & $10[\mathrm{DE}]$ & $8[2]$ & $8[30]$ & $3[15]$ & 0 [WS] & 5.8 [2-26-1] \\
\hline 7 & $\begin{array}{l}\text { Yemen Thrush } \\
\text { Turdus menachensis }\end{array}$ & 59.0 & $2.5[\mathrm{NT}]$ & 10 [DE] & $2.5[\mathrm{NT}]$ & $10[\mathrm{DE}]$ & $10[0.3]$ & 4 [5000] & $10[50]$ & $10[\mathrm{EA}]$ & 0 [87-176-6] \\
\hline 8 & $\begin{array}{l}\text { Arabian Grosbeak } \\
\text { Rhynchostruthus percivali }\end{array}$ & 58.8 & $2.5[\mathrm{NT}]$ & $10[\mathrm{DE}]$ & $2.5[\mathrm{NT}]$ & 10 [DE] & $8[2]$ & $6[500]$ & $4[20]$ & $10[\mathrm{EA}]$ & $5.8[3-211-1]$ \\
\hline 9 & $\begin{array}{l}\text { Arabian Bustard } \\
\text { Ardeotis arabs }\end{array}$ & 58.3 & $2.5[\mathrm{NT}]$ & 10 [DE] & $10[\mathrm{CR}]$ & $10[\mathrm{DE}]$ & $10[0]$ & $10[0]$ & $0[0]$ & 0 [WS] & 5.8 [2-26-1] \\
\hline 10 & $\begin{array}{l}\text { Northern Bald Ibis }{ }^{\dagger+} \\
\text { Geronticus eremita }\end{array}$ & 52.3 & $7.5[\mathrm{EN}]$ & $5[\mathrm{ST}]$ & $10[\mathrm{CR}]$ & $10[\mathrm{DE}]$ & $4[8]$ & $10[\mathrm{PE}]$ & $0[0]$ & 0 [WS] & $5.8[2-34-1]$ \\
\hline 11 & $\begin{array}{l}\text { Bearded Vulture }{ }^{\dagger} \\
\text { Gypaetus barbatus }\end{array}$ & 51.7 & $2.5[\mathrm{NT}]$ & $10[\mathrm{DE}]$ & $5[\mathrm{VU}]$ & $7.5[\mathrm{PD}]$ & $10[0]$ & $10[0]$ & $0[0]$ & 0 [WS] & $6.7[1-248-1]$ \\
\hline 12 & $\begin{array}{l}\text { Sooty Falcon } \\
\text { Falco concolor }\end{array}$ & 51.5 & $5[\mathrm{VU}]$ & 10 [DE] & $7.5[\mathrm{EN}]$ & $10[\mathrm{DE}]$ & $2[8]$ & $7[300]$ & $10[50]$ & 0 [WS] & 0 [39-64-7] \\
\hline 13 & $\begin{array}{l}\text { Crab Plover } \\
\text { Dromas ardeola }\end{array}$ & 51.0 & $0[\mathrm{LC}]$ & $5[\mathrm{ST}]$ & 5 [VU] & $10[\mathrm{DE}]$ & $6[4]$ & $5[1,400]$ & $10[50]$ & 0 [WS] & $10[1-1-1]$ \\
\hline 14 & $\begin{array}{l}\text { Common Ostrich }^{\dagger} \\
\text { Struthio camelus }\end{array}$ & 50.5 & $0[\mathrm{LC}]$ & 10 [DE] & $10[\mathrm{RE}]$ & $5[\mathrm{ST}]$ & $9[1]$ & $8[80]$ & $0.2[1]$ & 0 [WS] & $8.3[2-2-1]$ \\
\hline 15 & $\begin{array}{l}\text { Lappet-faced Vulture } \\
\text { Torgos tracheliotos }\end{array}$ & 49.7 & $7.5[\mathrm{EN}]$ & 10 [DE] & $5[\mathrm{VU}]$ & 7.5 [PD] & $0[33]$ & $6[500]$ & $2[10]$ & $5[\mathrm{ES}]$ & $6.7[1-248-1]$ \\
\hline 16 & $\begin{array}{l}\text { Arabian Waxbill } \\
\text { Estrilda rufibarba }\end{array}$ & 49.6 & $0[\mathrm{LC}]$ & 10 [DE] & $0[\mathrm{LC}]$ & 10 [DE] & $8[2]$ & $4[5,000]$ & $3.4[17]$ & $10[\mathrm{EA}]$ & 4.2 [16-141-1] \\
\hline 17 & $\begin{array}{l}\text { Tawny Eagle } \\
\text { Aquila rapax }\end{array}$ & 48.7 & $5[\mathrm{VU}]$ & 10 [DE] & $7.5[\mathrm{EN}]$ & 10 [DE] & 8 [2] & $7[100]$ & $0.4[2]$ & 0 [WS] & 0.8 [11-248-6] \\
\hline 18 & $\begin{array}{l}\text { Arabian Lark } \\
\text { Eremalauda eremodites }\end{array}$ & 48.3 & $0[\mathrm{LC}]$ & $5[\mathrm{ST}]$ & $0[\mathrm{LC}]$ & 10 [DE] & $0[58]$ & $3[17,000]$ & 17 [85] & 7.5 [NE] & 5.8 [2-92-1] \\
\hline 19 & $\begin{array}{l}\text { Cinereous Bunting }{ }^{\ddagger} \\
\text { Emberiza cineracea }\end{array}$ & 47.5 & $2.5[\mathrm{NT}]$ & 10 [DE] & $0[\mathrm{LC}]$ & $5[\mathrm{UN}]$ & $10[0.1]$ & $6[\mathrm{RA}]$ & 14 [70] & 0 [WS] & 0 [44-44-7] \\
\hline 20 & $\begin{array}{l}\text { Philby's Partridge }^{\dagger} \\
\text { Alectoris philbyi }\end{array}$ & 47.3 & $0[\mathrm{LC}]$ & $5[\mathrm{ST}]$ & $0[\mathrm{LC}]$ & $5[\mathrm{ST}]$ & $9[1]$ & $3[15,000]$ & $12[60]$ & $10[\mathrm{EA}]$ & 3.3 [7-182-3] \\
\hline 21 & $\begin{array}{l}\text { Collared Kingfisher }{ }^{\dagger} \\
\text { Todiramphus chloris }\end{array}$ & 47.2 & $0[\mathrm{LC}]$ & $10[\mathrm{DE}]$ & $7.5[\mathrm{EN}]$ & 10 [DE] & $9[1]$ & $7[300]$ & $0.4[2]$ & 0 [WS] & $3-3[25-119-1]$ \\
\hline 22 & $\begin{array}{l}\text { Red-billed Tropicbird } \\
\text { Phaethon aethereus }\end{array}$ & 47.2 & $0[\mathrm{LC}]$ & $10[\mathrm{DE}]$ & $0[\mathrm{LC}]$ & 7.5 [PD] & 8 [2] & $8[50]$ & $0.4[2]$ & 5 [ES] & $8.3[3-3-1]$ \\
\hline 23 & $\begin{array}{l}\text { Sociable Lapwing }{ }^{* \$} \\
\text { Vanellus gregarious }\end{array}$ & 47.0 & 10 [CR] & 10 [DE] & $10[\mathrm{CR}]$ & $5[\mathrm{UN}]$ & $4[2-47]$ & $6[\mathrm{RA}]$ & $2[10]$ & 0 [WS] & 0 [24-71-5] \\
\hline 24 & $\begin{array}{l}\text { Bateleur }{ }^{\$ 8} \\
\text { Terathopius ecaudatus }\end{array}$ & 46.7 & $2.5[\mathrm{NT}]$ & 10 [DE] & $2.5[\mathrm{NT}]$ & $5[\mathrm{UN}]$ & $10[0]$ & $10[0]$ & $0[0]$ & 0 [WS] & 6.7 [1-248-1] \\
\hline 25 & $\begin{array}{l}\text { Egyptian Vulture } \\
\text { Neophron percnopterus }\end{array}$ & 46.2 & $7.5[\mathrm{EN}]$ & 10 [DE] & $5[\mathrm{VU}]$ & 10 [DE] & $1[9]$ & 5 [1000] & $1[5]$ & 0 [WS] & $6.7[1-248-1]$ \\
\hline 26 & $\begin{array}{l}\text { European Goldfinch } \\
\text { Carduelis carduelis }\end{array}$ & 45.7 & $0[\mathrm{LC}]$ & 10 [DE] & $7.5[\mathrm{EN}]$ & 10 [DE] & $7[3]$ & $5[2000]$ & $0.4[2]$ & 0 [WS] & $5.8[4-211-1]$ \\
\hline 27 & $\begin{array}{l}\text { Great Knot }{ }^{t s} \\
\text { Calidris tenuirostris }\end{array}$ & 45.7 & $7.5[\mathrm{EN}]$ & 10 [DE] & $7.5[\mathrm{EN}]$ & $5[\mathrm{UN}]$ & $9.5[0.2-1]$ & $6[\mathrm{RA}]$ & $0.2[1]$ & 0 [WS] & 0 [24-91-8] \\
\hline 28 & $\begin{array}{l}\text { Yemen Linnet } \\
\text { Linaria yemenensis }\end{array}$ & 45.0 & $0[\mathrm{LC}]$ & $5[\mathrm{ST}]$ & $0[\mathrm{LC}]$ & $5[\mathrm{ST}]$ & $9[1]$ & $1[100,000]$ & $10[50]$ & $10[\mathrm{EA}]$ & 5 [4-211-2] \\
\hline 29 & $\begin{array}{l}\text { Helmeted Guineafowl } \\
\text { Numida meleagris }\end{array}$ & 44.5 & $0[\mathrm{LC}]$ & $5[\mathrm{ST}]$ & $5[\mathrm{VU}]$ & 10 [DE] & $10[0.5]$ & $6[500]$ & $0.2[1]$ & 0 [WS] & $8.3[1-8-1]$ \\
\hline
\end{tabular}


Avian Conservation and Ecology 15(2): 18

\begin{tabular}{|c|c|c|c|c|c|c|c|c|c|c|c|}
\hline 30 & $\begin{array}{l}\text { Arabian Serin }{ }^{\dagger} \\
\text { Crithagra rothschildi }\end{array}$ & 44.5 & $0[\mathrm{LC}]$ & $5[\mathrm{ST}]$ & $0[\mathrm{LC}]$ & $5[\mathrm{ST}]$ & 8 [2] & $1[260,000]$ & $13[65]$ & $10[\mathrm{EA}]$ & $2.5[25-211-2]$ \\
\hline 31 & $\begin{array}{l}\text { African Olive-Pigeon } \\
\text { Columba arquatrix }\end{array}$ & 43.2 & $0[\mathrm{LC}]$ & $10[\mathrm{DE}]$ & 7.5 [EN] & $5[\mathrm{ST}]$ & $10[0.1]$ & $8[75]$ & $1[5]$ & 0 [WS] & $1.7[35-350-3]$ \\
\hline 32 & $\begin{array}{l}\text { Hamerkop } \\
\text { Scopus umbretta }\end{array}$ & 42.4 & $0[\mathrm{LC}]$ & $5[\mathrm{ST}]$ & $5[\mathrm{VU}]$ & $10[\mathrm{DE}]$ & $7[3]$ & $5[1,500]$ & $0.4[2]$ & 0 [WS] & 10 [1-1-1] \\
\hline 33 & $\begin{array}{l}\text { Steppe Whimbree } \\
\text { Numenius p. alboaxillaris }\end{array}$ & 42.2 & $0[\mathrm{LC}]$ & $10[\mathrm{DE}]$ & $5[\mathrm{CR}] \mathrm{SS}$ & $5[\mathrm{UN}]$ & 4 [2-100] & 8 [ER] & $6[30]$ & 0 [WS] & 4.2 [8-91-2] \\
\hline 34 & $\begin{array}{l}\text { Greater Spotted Eagle } \\
\text { Clanga clanga }\end{array}$ & 41.8 & $5[\mathrm{VU}]$ & $10[\mathrm{DE}]$ & $5[\mathrm{VU}]$ & $5[\mathrm{UN}]$ & 8 [2] & $2[\mathrm{UC}]$ & $1[5]$ & 0 [WS] & 5.8 [3-248-1] \\
\hline 35 & $\begin{array}{l}\text { Pink-backed Pelican }{ }^{\dagger} \\
\text { Pelecanus rufescens }\end{array}$ & 41.8 & $0[\mathrm{LC}]$ & $5[\mathrm{ST}]$ & $5[\mathrm{VU}]$ & $10[\mathrm{DE}]$ & 8 [2] & $7[400]$ & $1[5]$ & 0 [WS] & $5.8[8-8-2]$ \\
\hline 36 & $\begin{array}{l}\text { Tristram's Starling } \\
\text { Onychognathus tristramii }\end{array}$ & 40.7 & $0[\mathrm{LC}]$ & $5[\mathrm{ST}]$ & $0[\mathrm{LC}]$ & $5[\mathrm{ST}]$ & $10[0.2]$ & $2[35,000]$ & $7[35]$ & $7.5[\mathrm{NE}]$ & $4.2[11-123-1]$ \\
\hline 37 & $\begin{array}{l}\text { Arabian Scops-Owl } \\
\text { Otus pamelae }\end{array}$ & 40.1 & $0[\mathrm{LC}]$ & $5[\mathrm{ST}]$ & $0[\mathrm{LC}]$ & $5[\mathrm{ST}]$ & $6[4]$ & $3[14,000]$ & $9.4[47]$ & 10 [EA] & $1.7[53-222-3]$ \\
\hline 38 & $\begin{array}{l}\text { (Arabian) Spotted Eagle-Owl" } \\
\text { Bubo africanus milesi }\end{array}$ & 39.8 & $0[\mathrm{LC}]$ & $5[\mathrm{ST}]$ & $0[\mathrm{LC}]$ & $5[\mathrm{ST}]$ & $5[5]$ & $6[800]$ & $8[40]$ & $7.5[\mathrm{PE}]$ & $3.3[18-222-2]$ \\
\hline 39 & $\begin{array}{l}\text { Buff-breasted Wheatear } \\
\text { Oenanthe bottae }\end{array}$ & 39.6 & $0[\mathrm{LC}]$ & $5[\mathrm{ST}]$ & $0[\mathrm{LC}]$ & $5[\mathrm{ST}]$ & $10[0.1]$ & $3[13,000]$ & $6.6[33]$ & $10[\mathrm{EA}]$ & 0 [28-335-14] \\
\hline 40 & $\begin{array}{l}\text { Saker Falcon } \\
\text { Falco cherrug }\end{array}$ & 39.5 & $7.5[\mathrm{EN}]$ & $10[\mathrm{DE}]$ & $10[\mathrm{CR}]$ & $5[\mathrm{UN}]$ & 0 [100-100] & $6[\mathrm{RA}]$ & $1[5]$ & 0 [WS] & 0 [39-64-7] \\
\hline 41 & $\begin{array}{l}\text { Harlequin Quail } \\
\text { Coturnix delegorguei }\end{array}$ & 38.9 & $0[\mathrm{LC}]$ & $5[\mathrm{ST}]$ & $2.5[\mathrm{NT}]$ & $5[\mathrm{UN}]$ & $10[0.5]$ & $7[300]$ & $0.2[1]$ & $5[\mathrm{ES}]$ & 4.2 [6-187-2] \\
\hline 42 & $\begin{array}{l}\text { Yemen Serin } \\
\text { Crithagra menachensis }\end{array}$ & 38.5 & $0[\mathrm{LC}]$ & $5[\mathrm{ST}]$ & $0[\mathrm{LC}]$ & $5[\mathrm{ST}]$ & $9[1]$ & $2[25,000]$ & $5[25]$ & $10[\mathrm{EA}]$ & $2.5[25-211-2]$ \\
\hline 43 & $\begin{array}{l}\text { Lanner Falcon } \\
\text { Falco biarmicus }\end{array}$ & 38.4 & $0[\mathrm{LC}]$ & $0[\mathrm{IN}]$ & $10[\mathrm{CR}]$ & $10[\mathrm{DE}]$ & $10[0]$ & $8[50]$ & $0.4[2]$ & 0 [WS] & 0 [39-64-7] \\
\hline 44 & $\begin{array}{l}\text { Short-toed Snake-Eagle } \\
\text { Circaetus gallicus }\end{array}$ & 38.0 & $0[\mathrm{LC}]$ & $5[\mathrm{ST}]$ & $5[\mathrm{VU}]$ & $5[\mathrm{ST}]$ & $9[1]$ & $7[120]$ & $2[10]$ & 0 [WS] & $5[6-248-1]$ \\
\hline 45 & $\begin{array}{l}\text { Arabian Sunbird } \\
\text { Cinnyris hellmayri }\end{array}$ & 35.5 & $0[\mathrm{LC}]$ & $5[\mathrm{ST}]$ & $0[\mathrm{LC}]$ & $5[\mathrm{ST}]$ & $2[8]$ & $1[250,000]$ & $10[50]$ & $10[\mathrm{EA}]$ & $2.5[58-147-2]$ \\
\hline 46 & $\begin{array}{l}\text { Pale Sparrow } \\
\text { Carpospiza brachydactyla }\end{array}$ & 35.2 & $0[\mathrm{LC}]$ & $5[\mathrm{ST}]$ & $0[\mathrm{LC}]$ & 2.5 [IN?] & 0 [19] & $5[2000]$ & $16[80]$ & 0 [WS] & $6.7[1-43-1]$ \\
\hline 47 & $\begin{array}{l}\text { Black-winged Kite } \\
\text { Elanus caeruleus }\end{array}$ & 35.0 & $0[\mathrm{LC}]$ & $5[\mathrm{ST}]$ & $5[\mathrm{VU}]$ & $0[\mathrm{IN}]$ & $10[0.5]$ & $9[3]$ & $0.2[1]$ & 0 [WS] & $5.8[4-248-1]$ \\
\hline 48 & $\begin{array}{l}\text { Dark Chanting-Goshawk } \\
\text { Melierax metabates }\end{array}$ & 35.0 & $0[\mathrm{LC}]$ & $5[\mathrm{ST}]$ & $0[\mathrm{LC}]$ & $5[\mathrm{ST}]$ & $8[2]$ & $6[750]$ & $0.2[1]$ & $5[\mathrm{ES}]$ & $5.8[3-248-1]$ \\
\hline 49 & $\begin{array}{l}\text { Brown Woodland-warbler } \\
\text { Phylloscopus umbrovirens }\end{array}$ & 35.0 & $0[\mathrm{LC}]$ & $5[\mathrm{ST}]$ & $0[\mathrm{LC}]$ & $10[\mathrm{DE}]$ & $9[1]$ & $2[40,000]$ & $4[20]$ & $5[\mathrm{ES}]$ & 0 [78-78-6] \\
\hline 50 & $\begin{array}{l}\text { Caspian Plover } \\
\text { Charadrius asiaticus }\end{array}$ & 35.0 & $0[\mathrm{LC}]$ & $10[\mathrm{DE}]$ & $0[\mathrm{LC}]$ & $5[\mathrm{UN}]$ & $0[48]$ & $4[\mathrm{SC}]$ & $16[80]$ & 0 [WS] & 0 [33-71-6] \\
\hline 51 & $\begin{array}{l}\text { Sand Partridge } \\
\text { Ammoperdix heyi }\end{array}$ & 35.0 & $0[\mathrm{LC}]$ & $5[\mathrm{ST}]$ & $0[\mathrm{LC}]$ & $10[\mathrm{DE}]$ & $0[48]$ & $0[530,000]$ & $10[50]$ & $5[\mathrm{ES}]$ & 5 [2-187-2] \\
\hline 52 & $\begin{array}{l}\text { Arabian Partridge }{ }^{\dagger} \\
\text { Alectoris melanocephala }\end{array}$ & 34.9 & $0[\mathrm{LC}]$ & $5[\mathrm{ST}]$ & $0[\mathrm{LC}]$ & $5[\mathrm{ST}]$ & $3[7]$ & $1[150,000]$ & $7.5[38]$ & $10[\mathrm{EA}]$ & $3.3[7-182-3]$ \\
\hline 53 & $\begin{array}{l}\text { Steppe Eagle }{ }^{* 8} \\
\text { Aquila nipalensis }\end{array}$ & 34.8 & $7.5[\mathrm{EN}]$ & $10[\mathrm{DE}]$ & $7.5[\mathrm{EN}]$ & $5[\mathrm{UN}]$ & 0 [100-100] & $0[\mathrm{CO}]$ & $4[20]$ & 0 [WS] & 0.8 [11-248-6] \\
\hline 54 & $\begin{array}{l}\text { Rufous-capped Lark } \\
\text { Calandrella eremica }\end{array}$ & 34.2 & $0[\mathrm{LC}]$ & $5[\mathrm{UN}]$ & $0[\mathrm{LC}]$ & $5[\mathrm{ST}]$ & $9[1]$ & $2[25,000]$ & $4[20]$ & $5[\mathrm{ES}]$ & 4.2 [6-92-2] \\
\hline 55 & $\begin{array}{l}\text { Goliath Heron } \\
\text { Ardea goliath }\end{array}$ & 32.5 & $0[\mathrm{LC}]$ & $5[\mathrm{ST}]$ & 7.5 [EN] & $5[\mathrm{UN}]$ & $6[4]$ & $8[60]$ & $0.2[1]$ & 0 [WS] & 0.8 [14-64-5] \\
\hline 56 & $\begin{array}{l}\text { Horsfield's Bushlark } \\
\text { Mirafra javanica }\end{array}$ & 32.0 & $0[\mathrm{LC}]$ & $5[\mathrm{ST}]$ & $0[\mathrm{LC}]$ & $2.5[\mathrm{PI}]$ & $9[1]$ & $7[200]$ & $0.2[1]$ & $5[\mathrm{ES}]$ & 3.3 [24-92-1] \\
\hline 57 & $\begin{array}{l}\text { Hypocolius } \\
\text { Hypocolius ampelinus }\end{array}$ & 32.0 & $0[\mathrm{LC}]$ & $5[\mathrm{UN}]$ & $0[\mathrm{LC}]$ & $5[\mathrm{ST}]$ & $0[50]$ & $0[\mathrm{CO}]$ & $12[60]$ & 0 [WS] & 10 [1-1-1] \\
\hline 58 & $\begin{array}{l}\text { Common Pochard }{ }^{8} \\
\text { Aythya ferina }\end{array}$ & 31.9 & $5[\mathrm{VU}]$ & 10 [DE] & $5[\mathrm{VU}]$ & $5[\mathrm{UN}]$ & 0 [63-100] & $4[\mathrm{SC}]$ & $0.4[2]$ & 0 [WS] & $2.5[12-165-3]$ \\
\hline 59 & $\begin{array}{l}\text { Arabian Golden Sparrow } \\
\text { Passer euchlorus }\end{array}$ & 31.7 & $0[\mathrm{LC}]$ & $5[\mathrm{ST}]$ & $0[\mathrm{LC}]$ & $5[\mathrm{ST}]$ & $8[2]$ & $2[25,000]$ & $10[50]$ & 0 [WS] & $1.7[28-43-3]$ \\
\hline 60 & $\begin{array}{l}\text { Griffon Vulture } \\
\text { Gyps fulvus }\end{array}$ & 31.7 & $0[\mathrm{LC}]$ & $0[\mathrm{IN}]$ & 7.5 [EN] & $10[\mathrm{DE}]$ & $2[8]$ & $5[2500]$ & $3[15]$ & 0 [WS] & $4.2[8-248-2]$ \\
\hline 61 & $\begin{array}{l}\text { Egyptian Nightjar } \\
\text { Caprimulgus aegyptius }\end{array}$ & 31.5 & $0[\mathrm{LC}]$ & 10 [DE] & $0[\mathrm{LC}]$ & $5[\mathrm{ST}]$ & $2.5[5-52]$ & $2[\mathrm{UC}]$ & $12[60]$ & 0 [WS] & 0 [38-98-5] \\
\hline 62 & $\begin{array}{l}\text { Sinai Rosefinch } \\
\text { Carpodacus synoicus }\end{array}$ & 31.5 & $0[\mathrm{LC}]$ & $5[\mathrm{ST}]$ & $0[\mathrm{LC}]$ & $5[\mathrm{ST}]$ & $3[7]$ & $4[5000]$ & $12[60]$ & 0 [WS] & $2.5[25-211-2]$ \\
\hline 63 & $\begin{array}{l}\text { African Paradise-Flycatcher } \\
\text { Terpsiphone viridis }\end{array}$ & 31.4 & $0[\mathrm{LC}]$ & $5[\mathrm{ST}]$ & $0[\mathrm{LC}]$ & $5[\mathrm{ST}]$ & $7[3]$ & $5[1500]$ & $0.2[1]$ & $5[\mathrm{ES}]$ & $4.2[17-105-1]$ \\
\hline 64 & $\begin{array}{l}\text { African Pipit } \\
\text { Anthus cinnamomeus }\end{array}$ & 31.2 & $0[\mathrm{LC}]$ & $5[\mathrm{ST}]$ & $0[\mathrm{LC}]$ & $5[\mathrm{ST}]$ & $9[1]$ & $7[100]$ & $0.2[1]$ & $5[\mathrm{ES}]$ & 0 [41-67-7] \\
\hline 65 & $\begin{array}{l}\text { Brown Noddy } \\
\text { Anous stolidus }\end{array}$ & 31.0 & $0[\mathrm{LC}]$ & $5[\mathrm{ST}]$ & $0[\mathrm{LC}]$ & $5[\mathrm{UN}]$ & $6[4]$ & 4 [7200] & $0.2[1]$ & $5[\mathrm{ES}]$ & $5.8[5-100-1]$ \\
\hline 66 & $\begin{array}{l}\text { White-eyed Gull } \\
\text { Larus leucophthalmus }\end{array}$ & 31.0 & $0[\mathrm{LC}]$ & $5[\mathrm{ST}]$ & $0[\mathrm{LC}]$ & $5[\mathrm{ST}]$ & $4[6]$ & $5[3000]$ & $12[60]$ & 0 [WS] & 0 [29-33-7] \\
\hline
\end{tabular}


Avian Conservation and Ecology 15(2): 18

http://www.ace-eco.org/vol15/iss2/art18/

\begin{tabular}{|c|c|c|c|c|c|c|c|c|c|c|c|}
\hline 67 & $\begin{array}{l}\text { Black-crowned Tchagra } \\
\text { Tchagra senegalus }\end{array}$ & 30.5 & $0[\mathrm{LC}]$ & $5[\mathrm{ST}]$ & $0[\mathrm{LC}]$ & 2.5 [IN?] & $7[3]$ & $5[2000]$ & $0.2[1]$ & $5[\mathrm{ES}]$ & $5.8[4-48-1]$ \\
\hline 68 & $\begin{array}{l}\text { Golden Eagle } \\
\text { Aquila chrysaetos }\end{array}$ & 30.5 & $0[\mathrm{LC}]$ & $5[\mathrm{ST}]$ & $7.5[\mathrm{EN}]$ & $10[\mathrm{DE}]$ & $0[12]$ & 7 [200] & $0.2[1]$ & 0 [WS] & $0.8[11-248-6]$ \\
\hline 69 & $\begin{array}{l}\text { Grey-headed Kingfisher } \\
\text { Halcyon leucocephala }\end{array}$ & 30.5 & $0[\mathrm{LC}]$ & $5[\mathrm{ST}]$ & $0[\mathrm{LC}]$ & $5[\mathrm{UN}]$ & $7[3]$ & $5[1500]$ & $0.2[1]$ & $5[\mathrm{ES}]$ & $3.3[12-120-2]$ \\
\hline 70 & $\begin{array}{l}\text { Kurdish Wheatear }{ }^{\ddagger} \\
\text { Oenanthe xanthoprymna }\end{array}$ & 30.5 & $0[\mathrm{LC}]$ & $5[\mathrm{ST}]$ & $0[\mathrm{LC}]$ & $5[\mathrm{UN}]$ & $2.5[5-100]$ & $6[\mathrm{RA}]$ & $12[60]$ & 0 [WS] & 0 [28-335-14] \\
\hline 71 & $\begin{array}{l}\text { Upcher's Warbler } \\
\text { Hippolais languida }\end{array}$ & 30.2 & $0[\mathrm{LC}]$ & $5[\mathrm{ST}]$ & $0[\mathrm{LC}]$ & $5[\mathrm{ST}]$ & $0[100]$ & $2[\mathrm{UC}]$ & 14 [70] & 0 [WS] & $4.2[4-54-3]$ \\
\hline 72 & $\begin{array}{l}\text { Arabian Warbler } \\
\text { Sylvia leucomelaena }\end{array}$ & 30.0 & $0[\mathrm{LC}]$ & $10[\mathrm{DE}]$ & $0[\mathrm{LC}]$ & $5[\mathrm{ST}]$ & $0[13]$ & $2[65,000]$ & $8[40]$ & $5[\mathrm{ES}]$ & 0 [30-69-13] \\
\hline 73 & $\begin{array}{l}\text { Mourning Wheatear } \\
\text { Oenanthe lugens }\end{array}$ & 30.0 & $0[\mathrm{LC}]$ & $5[\mathrm{ST}]$ & $0[\mathrm{LC}]$ & $10[\mathrm{DE}]$ & $0[14]$ & $0[445,000]$ & $10[50]$ & $5[\mathrm{ES}]$ & 0 [28-335-14] \\
\hline 74 & $\begin{array}{l}\text { Common Stonechat } \\
\text { Saxicola torquatus }\end{array}$ & 29.7 & $0[\mathrm{LC}]$ & $5[\mathrm{ST}]$ & $0[\mathrm{LC}]$ & $5[\mathrm{ST}]$ & $8[2]$ & $3[20,000]$ & 0.4 [2] & $5[\mathrm{ES}]$ & $3.3[11-335-2]$ \\
\hline 75 & $\begin{array}{l}\text { Streaked Scrub-Warbler } \\
\text { Scotocerca inquieta }\end{array}$ & 29.7 & $0[\mathrm{LC}]$ & $10[\mathrm{DE}]$ & $0[\mathrm{LC}]$ & $5[\mathrm{ST}]$ & $0[43]$ & $0[300,000]$ & $3[15]$ & $5[\mathrm{ES}]$ & $6.7[1-37-1]$ \\
\hline 76 & $\begin{array}{l}\text { Arabian Babbler } \\
\text { Argya squamiceps }\end{array}$ & 29.5 & $0[\mathrm{LC}]$ & $0[\mathrm{IN}]$ & $0[\mathrm{LC}]$ & $0[\mathrm{IN}]$ & $0[37]$ & $2[75,000]$ & $10[50]$ & $7.5[\mathrm{NE}]$ & 5 [9-148-1] \\
\hline 77 & $\begin{array}{l}\text { Desert Tawny Owl }{ }^{\dagger} \\
\text { Strix hadorami }\end{array}$ & 29.2 & $0[\mathrm{LC}]$ & $5[\mathrm{ST}]$ & $0[\mathrm{LC}]$ & $5[\mathrm{ST}]$ & $0[19]$ & 5 [1800] & $10[50]$ & $0[\mathrm{WS}]$ & $4.2[17-222-1]$ \\
\hline 78 & $\begin{array}{l}\text { Little Rock-Thrush } \\
\text { Monticola rufocinereus }\end{array}$ & 29.0 & $0[\mathrm{LC}]$ & $5[\mathrm{ST}]$ & $0[\mathrm{LC}]$ & $2.5[\mathrm{PI}]$ & $9[1]$ & $3[12,500]$ & $2[10]$ & $5[\mathrm{ES}]$ & $2.5[14-335-3]$ \\
\hline 79 & $\begin{array}{l}\text { Red-tailed Shrike } e^{\ddagger} \\
\text { Lanius phoenicuroides }\end{array}$ & 29.0 & $0[\mathrm{LC}]$ & $5[\mathrm{ST}]$ & $0[\mathrm{LC}]$ & $5[\mathrm{ST}]$ & 3 [4-100] & $0[\mathrm{CO}]$ & $16[80]$ & 0 [WS] & 0 [29-33-7] \\
\hline 80 & $\begin{array}{l}\text { Eastern Imperial Eagle }{ }^{\dagger+\$ 8} \\
\text { Aquila heliaca }\end{array}$ & 28.8 & $5[\mathrm{VU}]$ & $10[\mathrm{DE}]$ & $5[\mathrm{VU}]$ & $5[\mathrm{UN}]$ & $0[49]$ & $2[\mathrm{UC}]$ & $1[5]$ & 0 [WS] & 0.8 [11-248-6] \\
\hline 81 & $\begin{array}{l}\text { Nubian Nightjar } \\
\text { Caprimulgus nubicus }\end{array}$ & 28.5 & $0[\mathrm{LC}]$ & $10[\mathrm{DE}]$ & $0[\mathrm{LC}]$ & $5[\mathrm{ST}]$ & $5[5]$ & $5[2400]$ & $1[5]$ & $2.5[\mathrm{NS}]$ & 0 [38-98-5] \\
\hline 82 & $\begin{array}{l}\text { Black-bellied Sandgrouse } \\
\text { Pterocles orientalis }\end{array}$ & 28.4 & $0[\mathrm{LC}]$ & $10[\mathrm{DE}]$ & $7.5[\mathrm{EN}]$ & $5[\mathrm{UN}]$ & $0[22]$ & $4[\mathrm{SC}]$ & $0.2[1]$ & 0 [WS] & 1.7 [14-16-6] \\
\hline 83 & $\begin{array}{l}\text { Abyssinian White-eye } \\
\text { Zosterops abyssinicus }\end{array}$ & 28.3 & $0[\mathrm{LC}]$ & $5[\mathrm{UN}]$ & $0[\mathrm{LC}]$ & $5[\mathrm{ST}]$ & $6[4]$ & $1[200,000]$ & $3[15]$ & $5[\mathrm{ES}]$ & $3.3[49-135-1]$ \\
\hline 84 & $\begin{array}{l}\text { European Turtle-Dove } \\
\text { Streptopelia turtur }\end{array}$ & 27.8 & $5[\mathrm{VU}]$ & 10 [DE] & $0[\mathrm{LC}]$ & $5[\mathrm{UN}]$ & $0[26]$ & 5 [1600] & $2[10]$ & 0 [WS] & $0.8[15-350-5]$ \\
\hline 85 & $\begin{array}{l}\text { Verreaux's Eagle } \\
\text { Aquila verreauxii }\end{array}$ & 27.7 & $0[\mathrm{LC}]$ & $5[\mathrm{ST}]$ & $7.5[\mathrm{EN}]$ & $0[\mathrm{IN}]$ & $6[4]$ & $8[25]$ & 0.4 [2] & 0 [WS] & $0.8[11-248-6]$ \\
\hline 86 & $\begin{array}{l}\text { Crested Lark } \\
\text { Galerida cristata }\end{array}$ & 26.0 & $0[\mathrm{LC}]$ & 10 [DE] & $0[\mathrm{LC}]$ & $5[\mathrm{ST}]$ & $0[82]$ & $0[4,000,000]$ & $1[5]$ & $5[\mathrm{ES}]$ & 5 [6-92-1] \\
\hline 87 & $\begin{array}{l}\text { Ménétries's Warbler } \\
\text { Sylvia mystacea }\end{array}$ & 26.0 & $0[\mathrm{LC}]$ & $5[\mathrm{ST}]$ & $0[\mathrm{LC}]$ & $5[\mathrm{UN}]$ & 0 [43-100] & $2[\mathrm{UC}]$ & 14 [70] & 0 [WS] & 0 [30-69-13] \\
\hline 88 & $\begin{array}{l}\text { Arabian Green Bee-eater } \\
\text { Merops cyanophrys }\end{array}$ & 25.3 & $0[\mathrm{LC}]$ & $0[\mathrm{IN}]$ & $0[\mathrm{LC}]$ & $0[\mathrm{IN}]$ & $0[36]$ & $2[75,000]$ & $10[50]$ & $7.5[\mathrm{NE}]$ & 0.8 [28-31-4] \\
\hline 89 & $\begin{array}{l}\text { Levant Sparrowhawk }{ }^{\mathrm{k}} \\
\text { Accipiter brevipes }\end{array}$ & 24.8 & $0[\mathrm{LC}]$ & $5[\mathrm{ST}]$ & $0[\mathrm{LC}]$ & $5[\mathrm{UN}]$ & $0[24]$ & $4[\mathrm{SC}]$ & $10[50]$ & 0 [WS] & 0.8 [48-248-4] \\
\hline 90 & $\begin{array}{l}\text { Long-billed Pipit } \\
\text { Anthus similis }\end{array}$ & 24.4 & $0[\mathrm{LC}]$ & $5[\mathrm{ST}]$ & $0[\mathrm{LC}]$ & $5[\mathrm{ST}]$ & $6[4]$ & $3[20,000]$ & $0.4[2]$ & 5 [ES] & $0[41-67-7]$ \\
\hline 91 & $\begin{array}{l}\text { Fan-tailed Raven } \\
\text { Corvus rhipidurus }\end{array}$ & 24.2 & $0[\mathrm{LC}]$ & 10 [DE] & $0[\mathrm{LC}]$ & $5[\mathrm{ST}]$ & $0[38]$ & $2[90,000]$ & $3[15]$ & $2.5[\mathrm{NS}]$ & $1.7[43-130-3]$ \\
\hline 92 & $\begin{array}{l}\text { Chestnut-bellied Sandgrouse }{ }^{\dagger} \\
\text { Pterocles exustus }\end{array}$ & 24.1 & $0[\mathrm{LC}]$ & $5[\mathrm{ST}]$ & $0[\mathrm{LC}]$ & $5[\mathrm{ST}]$ & $4[6]$ & $3[20,000]$ & 0.4 [2] & $5[\mathrm{ES}]$ & 1.7 [14-16-6] \\
\hline 93 & $\begin{array}{l}\text { Pied Wheatear } \\
\text { Oenanthe pleschanka }\end{array}$ & 22.0 & $0[\mathrm{LC}]$ & $5[\mathrm{ST}]$ & $0[\mathrm{LC}]$ & $5[\mathrm{UN}]$ & $0[100]$ & $0[\mathrm{CO}]$ & $12[60]$ & $0[\mathrm{WS}]$ & $0[28-335-14]$ \\
\hline 94 & $\begin{array}{l}\text { Graceful Prinia } \\
\text { Prinia gracilis }\end{array}$ & 21.3 & $0[\mathrm{LC}]$ & $5[\mathrm{ST}]$ & $0[\mathrm{LC}]$ & $5[\mathrm{ST}]$ & $0[27]$ & $2[45,000]$ & $1[5]$ & $5[\mathrm{ES}]$ & $3.3[26-161-1]$ \\
\hline 95 & $\begin{array}{l}\text { Cinnamon-breasted Bunting } \\
\text { Emberiza tahapisi }\end{array}$ & 21.2 & $0[\mathrm{LC}]$ & $5[\mathrm{ST}]$ & $0[\mathrm{LC}]$ & $5[\mathrm{ST}]$ & $6[4]$ & $0[350,000]$ & $0.2[1]$ & $5[\mathrm{ES}]$ & $0[44-44-7]$ \\
\hline 96 & $\begin{array}{l}\text { Peregrine Falcon } \\
\text { Falco peregrinus }\end{array}$ & 21.2 & $0[\mathrm{LC}]$ & $5[\mathrm{ST}]$ & $7.5[\mathrm{EN}]$ & $2.5[\mathrm{PI}]$ & $0[29]$ & $6[500]$ & $0.2[1]$ & 0 [WS] & 0 [39-64-7] \\
\hline 97 & $\begin{array}{l}\text { House Sparrow } \\
\text { Passer domesticus hufufae }\end{array}$ & 20.2 & $0[\mathrm{LC}]$ & 10 [DE] & $0[\mathrm{LC}]$ & $2.5[\mathrm{PI}]$ & $0[78]$ & $0[4,000,000]$ & $1[5]$ & 5 [ES] & 1.7 [28-43-3] \\
\hline 98 & $\begin{array}{l}\text { Isabelline Wheatear } \\
\text { Oenanthe isabellina }\end{array}$ & 20.0 & $0[\mathrm{LC}]$ & $5[\mathrm{ST}]$ & $0[\mathrm{LC}]$ & $5[\mathrm{ST}]$ & 0 [100-100] & $0[\mathrm{CO}]$ & $10[50]$ & 0 [WS] & 0 [28-335-14] \\
\hline 99 & $\begin{array}{l}\text { Black Scrub-Robin } \\
\text { Cercotrichas podobe }\end{array}$ & 15.5 & $0[\mathrm{LC}]$ & $5[\mathrm{ST}]$ & $0[\mathrm{LC}]$ & $0[\mathrm{IN}]$ & $0[42]$ & $1[250,000]$ & $2[10]$ & $2.5[\mathrm{NS}]$ & 5 [5-335-2] \\
\hline 100 & $\begin{array}{l}\text { White-crowned Wheatear } \\
\text { Oenanthe leucopyga }\end{array}$ & 15.5 & $0[\mathrm{LC}]$ & $5[\mathrm{ST}]$ & $0[\mathrm{LC}]$ & $5[\mathrm{ST}]$ & 0 [49] & $0[300,000]$ & $3[15]$ & $2.5[\mathrm{NS}]$ & 0 [28-335-14] \\
\hline 101 & $\begin{array}{l}\text { Blackstart } \\
\text { Oenanthe melanura }\end{array}$ & 14.0 & $0[\mathrm{LC}]$ & $5[\mathrm{ST}]$ & $0[\mathrm{LC}]$ & $0[\mathrm{IN}]$ & 0 [41] & $0[900,000]$ & $4[20]$ & $5[\mathrm{ES}]$ & 0 [28-335-14] \\
\hline 102 & $\begin{array}{l}\text { White-spectacled Bulbul } \\
\text { Pycnonotus xanthopygos }\end{array}$ & 12.5 & $0[\mathrm{LC}]$ & $0[\mathrm{IN}]$ & $0[\mathrm{LC}]$ & $0[\mathrm{IN}]$ & $0[33]$ & $0[1,500,000]$ & $10[50]$ & 0 [WS] & $2.5[41-157-2]$ \\
\hline
\end{tabular}

endemic to the region, four are near endemic, and one (Arabian Spotted Eagle-Owl) is probably endemic to the Arabian Peninsula. Thirty-four species contain subspecies that are endemic ( $n=30$ species) or near endemic ( $n=4$ species) to the Arabian Peninsula (Table 2). Thirty-three species were included because more than $50 \%$ of the global population is likely to occur 
in Saudi Arabia. Finally the Whimbrel(Numenius phaeopus) made the list because of the critically endangered status of the subspecies "Steppe Whimbrel." The final list consists of 81 breeding species and 21 nonbreeding species. The overall conservation scores of the 102 high conservation priority species ranged from a high of 87.8 (out of a possible 100) for the Asir Magpie (Pica asirensis) to a low of 12.5 for the White-spectacled Bulbul (Pycnonotus xanthopygos; Fig. 1, Table 2).

Fig. 1. Frequency histogram of the conservation priority scores for all 102 high conservation priority bird species in Saudi Arabia.

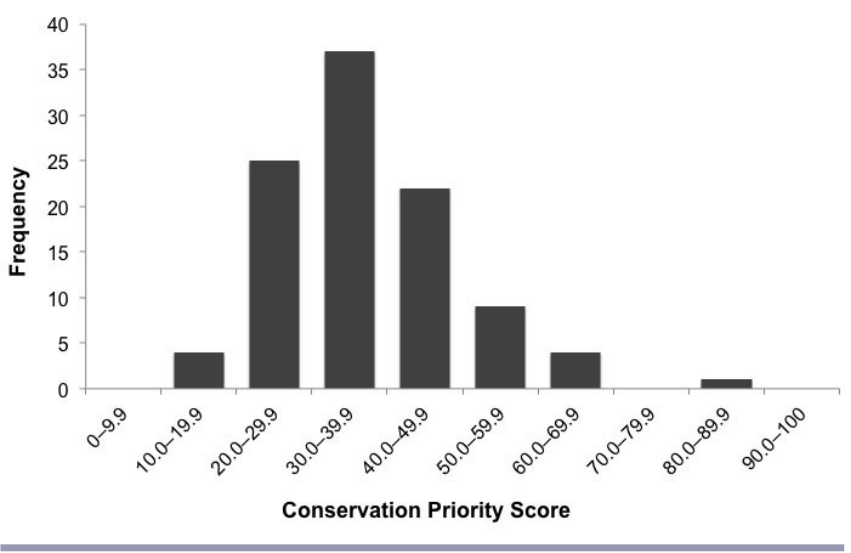

\section{Mapping the high conservation priority species}

Higher concentrations of high conservation priority species are located in the Asir Mountains and foothills in southwestern Saudi Arabia (Fig. 2), followed by the Red Sea coast, and the Tihama coastal plains (between the Red Sea coast and the Asir Mountains). The point with the highest high conservation priority score was $18 \mathrm{~km} \mathrm{WNW}$ of Abha city $\left(18.251406^{\circ} \mathrm{N}, 42.344104^{\circ}\right.$ E) in the vicinity of Jabal Sawda' just inside the Asir National Park. The $100-\mathrm{km}^{2}$ patch with the highest average conservation priority score was between Tanumah and Al-Ithnayn in the Asir Mountains, not within any existing protected area. The lowest concentrations of high conservation priority species occurred in the Rub' al-Khali, Great Nafud, and Ad-Dahna deserts. The location with the lowest overall score was in the Rub' al-Khali along the Yemen border around Sharorah (around $17.583333^{\circ} \mathrm{N}$, $47.416666^{\circ} \mathrm{E}$ ) and the surrounding area comprised the $100-\mathrm{km}^{2}$ patch with the lowest average conservation priority score (Fig. 2).

\section{DISCUSSION}

\section{Ranking the high conservation priority species}

Our analysis has more than doubled the number of high conservation priority bird species in Saudi Arabia from 49 in 2003 when the first list was developed (NCWCD 2003) to 102 in 2020. This significant increase is due to a better understanding of bird distribution and abundance, revised taxonomy, revised global assessments, and new regional assessments since the NCWCD produced their list in 2003. We then developed a relatively objective scoring system to rank Saudi Arabia's high conservation priority species to help draw attention to key species both within the Kingdom and the Arabian Peninsula in general.

Fig. 2. Heat map of the summed conservation scores for all high conservation priority bird species per location across Saudi Arabia. Locations with higher summed conservation priority scores are depicted in redder colors, whereas locations with lower scores are shown in bluer colors. The yellow circle denotes the individual point with the highest summed conservation priority score. The blue circle represents the 100$\mathrm{km}^{2}$ patch with the highest average summed conservation priority scores. The purple circle represents both the point with the lowest summed conservation priority score and the $100-\mathrm{km}^{2}$ patch with the lowest average summed conservation priority scores. Black dots represent provincial capitals.

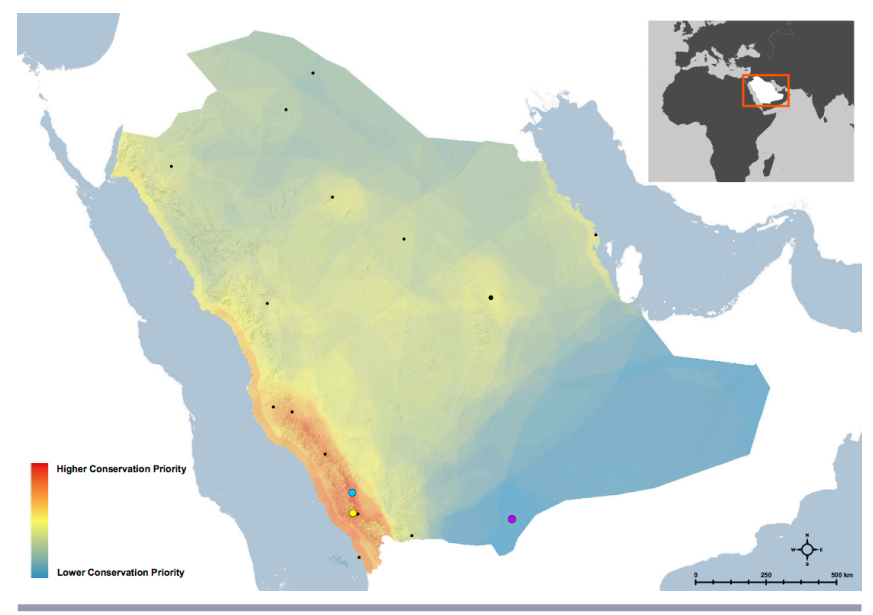

There is no standardized method for ranking national conservation priorities for birds or any other taxa. Most prioritization schemes rank species according to extinction risk along with various measures of species value, such as degree of national responsibility, endemicity, evolutionary distinctiveness, and social significance (Schmeller et al. 2008, Le Berre et al. 2019). We discuss our approach to each of these criteria below.

To assess each species' extinction risk, we scored conservation status and population trend at both the regional and global levels using IUCN Red List data (Symes et al. 2015, IUCN 2020). To measure national extinction risk, we quantified and scored each species' area of occurrence and relative abundance. Species that are restricted to a smaller area and/or in lower densities are typically more prone to impacts from deleterious environmental changes and catastrophic or stochastic events and therefore scored higher.

To measure Saudi Arabia's level of responsibility for each species, birds were scored according to their levels of endemicity and the proportion of the global population that occurs within the Kingdom at some stage during the year. The latter is an estimate of the contribution of the local population to the global survival of the species and as such is an important indicator of a species' conservation risk within a given nation (Schmeller et al. 2008, 2014, Kukkala et al. 2019). 
Each species was scored for its degree of evolutionary distinctiveness, which is a gauge of a species' isolation on its phylogenetic tree and thus a measure of its contribution to the total evolutionary history of its clade (Bennett et al. 2014, Jetz et al. 2014). This is an important consideration in conservation because the loss of a species in an old, monotypic, or species-poor clade would result in a greater loss of biodiversity than that of a young species with many close relatives (Isaac et al. 2007). Our scoring system also included a measure of distinctiveness within Saudi Arabia to prioritize species that contribute to phylogenetic diversity within the Kingdom.

Several prioritization schemes include social attributes such as cultural significance as a measure for determining conservation priority (e.g., Mace et al. 2007, Vieira da Silva et al. 2016); however this attribute was not included in our analysis because of its intrinsically subjective nature. Certainly some species have particular cultural relevance in Saudi Arabia and the Arabian Peninsula in general. For example, Saker (Falco cherrug), Lanner (F. biarmicus), and Peregrine Falcons (F. peregrinus) as well as Golden Eagles (Aquila chrysaetos) are widely esteemed for their use in traditional falconry, and to a lesser extent so are Asian Houbara (Chlamydotis macqueenii), Arabian Bustard, Common Quail (Coturnix coturnix), and Eurasian Thick-knee (Burhinus oedicnemus) for their use as quarry. The long history of falconry in Arabia underpinned UNESCO's decision in 2012 to inscribe falconry on the list of humanity's Intangible Cultural Heritage. But is the traditional use of trained birds a sufficient reason to elevate a species conservation priority over other species, which may be in dire need of protection? Indeed some could take exception to elevating the status of falcons on the basis that for generations they have been used to kill other birds, including species that are now critically endangered (Asian Houbara) or possibly extinct (Arabian Bustard) in the region. Certainly conservation biologists and land managers would be wise to highlight a bird's cultural value when garnering support to protect a high conservation priority species (Bowen-Jones and Entwistle 2002, Jepson and Barua 2015). However, using cultural value to rank a species' conservation priority against another species is wrought with subjectivity.

Other conservation prioritization schemes have used subjective criteria to rank birds very effectively, such as a species' value as a flagship, umbrella, or indicator species (Lambeck 1997, Simberloff 1998, Lindenmayer et al. 2000, Roberge et al. 2008, McGowan et al. 2020), or its scientific value, beauty, or charisma (Mace and Hudson 1999, Small 2011, Ducarme et al. 2013). However, because there are no data available for these attributes within Saudi Arabia they were excluded from our analysis.

Our scoring system gives higher overall scores to species that (i) have a poorer global conservation status and trend, (ii) have a poorer regional conservation status and trend, (iii) have lower numbers of breeding, or wintering and migrating birds within Saudi Arabia, (iv) have a greater proportion of the global population in or passing through Saudi Arabia, (v) occur within a smaller portion of Saudi Arabia, (vi) are regionally endemic, and (vii) have fewer species within their genera and family. The worst-case scenario (a species that is critically endangered and decreasing both globally and regionally, endemic to Saudi Arabia but possibly extinct, and the only member of its genera and family) would score the maximum score of 100 . Conversely the best-case scenario (a species that is of least concern and increasing both globally and regionally, with more than 300,000 breeding pairs in the Kingdom, is not endemic to Arabia, comprises less than 1\% of the global population, and is a member of a family and genus with dozens of species) would score 0 .

Unlike most other prioritization schemes, our methodology considers both breeding and nonbreeding species. Overall, the list includes 81 breeding species, which represents $37 \%$ of the 219 species that have been recorded breeding in the Kingdom (Boland and Alsuhaibany 2020). The nine highest conservation priority species all breed within Saudi Arabia, while the highest ranked nonbreeding species is the Northern Bald Ibis (Geronticus eremita; 10). Regional endemic species fill nine of the top 20 rankings (including six of the top eight).

The Asir Magpie was ranked as the bird species of highest conservation priority within Saudi Arabia, by a considerable margin. Its high ranking is due to its endangered status (both globally and regionally), its small and decreasing population size (perhaps 100 pairs), small range (around $3000 \mathrm{~km}^{2}$ ), and the fact that its global population occurs entirely within the Kingdom. The Asir Magpie's ranking underlines the need to mount an urgent conservation program to protect and restore the species, which to date has received almost no specific conservation effort (Boland and Alsuhaibany 2020). It is the Kingdom's only nationally endemic bird, occurs within Arabia's only endemic biodiversity hotspot (Conservation International 2020), and has rich potential to be the national bird of Saudi Arabia.

Our ranking system also draws attention to the importance of Saudi Arabia to the conservation of the globally endangered Basra Reed-Warbler (Acrocephalus griseldis), which ranked second according to our methodology. More than $90 \%$ of the global population is likely to pass through the Kingdom on migration each year, while perhaps 10 pairs breed in spring along the Riyadh River (an artificial wetland). As far as we are aware, this species has not received any conservation attention within Saudi Arabia and was not included in the original NCWCD list of high conservation priority species in 2003. Other species that were not included in the 2003 list that nonetheless ranked among the 20 highest conservation priority birds are the Tawny Eagle (Aquila rapax; 17), Arabian Lark (Eremalauda eremodites; 18), and Cinereous Bunting (Emberiza cineracea; 19).

Three of the 10 highest ranked species are in imminent danger of extinction within Saudi Arabia: Asian Houbara (6), Arabian Bustard (9), and Northern Bald Ibis (10); indeed the latter two may already be extinct within the Kingdom. As noted, the Asir Magpie (1) appears to be in significant peril as well, while the Common Ostrich (Struthio camelus; 15) went extinct across Arabia in the 20th century and has since been reintroduced into fenced areas within the Kingdom. Therefore our scoring system identified some of the more obvious species of conservation concern, as well as species that tend to be overlooked (such as the Basra Reed-Warbler, Tawny Eagle, Arabian Lark, and Cinereous Bunting). Of the 20 highest ranked species only the Asian Houbara (6) has received much or any conservation attention within Saudi Arabia (e.g., Islam et al. 2013, Nabi et al. 2019). 
Our ranking system draws attention to high conservation priority species within Saudi Arabia. Agencies, private landowners, and industries responsible for biodiversity conservation can use this list (and Fig. 2) to help inform overall conservation priorities within the Kingdom. Certainly other factors need to be incorporated in a conservation decision matrix, such as the cost of species' management, the likelihood that management intervention will succeed, habitat vulnerability, and the urgency of species decline (McCarthy et al. 2008, Joseph et al. 2009). Nonetheless, this list provides an important starting point for conservation agencies.

\section{Limitations of the species prioritization system}

Although we have attempted to create an objective, empirical measure of species conservation priority, there is nonetheless some subjectivity inherent in the scoring system. For example, one criterion for inclusion on the high conservation priority list is whether $50 \%$ or more of a species' global population occurs within Saudi Arabia at some stage during the year. However, that threshold could be lowered to, say, 30\%. Ultimately a somewhat pragmatic decision was made to set the threshold at $50 \%$. Incidentally, lowering the threshold to $30 \%$ would add an extra 26 species to the lower end of list; but it is unlikely that speciesspecific conservation actions would be taken on species ranked so far down the list.

Another limitation in our scoring system (which applies to most prioritization systems) is the subjective weighting applied to various attributes. For example, should regional conservation status be given more or less weight than global conservation status in a national prioritization scheme? Regional and global statuses are neither completely independent nor dependent on one another to a consistent extent across species (Wells et al. 2010), which perhaps weakens the scoring system. For this reason we ranked global and regional statuses equally. Similarly, is the number of breeding pairs of a resident species as important as abundance for a migratory species (which is the approach we took here)? Ultimately the weighting system is arbitrary but applies equally to all species.

In addition, we estimated the percentage of the global population that occurs within Saudi Arabia based on species range maps under the assumption that species are distributed uniformly across their range. This assumption is obviously flawed: for instance, traditional staging sites are essential for the survival of migratory species, but these are not distributed uniformly within or between countries; some species may forage over vast areas whereas others (such as wetland birds) might utilize only a small fraction of their range map; some migratory species may avoid flying over Saudi Arabia entirely, others may prefer it, and so on. Likewise, there was no attempt to account for variation in geographic marginality within a species range. For example, some parts of a species' range may be of more conservation value than others: central parts of a range or isolated outposts might secure future refugia, genetic diversity, and/or evolutionary potential (Schnittler and Günther 1999, Kukkala et al. 2019). However, in the absence of these data, which could take decades to acquire, this scoring system serves as a reasonably objective method for quickly ranking the conservation priorities of birds in poorly studied regions.

\section{Mapping the high conservation priority species}

Our GIS analysis revealed that the areas of highest conservation priority occur in the southwest of Saudi Arabia, particularly in the Asir Mountains and west into the Asir foothills, Tihama coastal plains, and Red Sea coast (Fig. 2). This result remained consistent regardless of whether the total count of high conservation priority species was mapped or the overall conservation scores for each species was summed and mapped. The Asir Mountains contain 16 of the 20 Arabian endemic, near endemic, or "probably endemic" species that occur within Saudi Arabia, and the greatest bird diversity in the Kingdom (Boland and Alsuhaibany 2020). Furthermore, Saudi Arabia's only nationally endemic bird species (and the species of highest conservation priority), the Asir Magpie, is entirely confined to these mountains. Conversely, the extreme desert regions of Saudi Arabia (the Rub' al-Khali, Great Nafud, and ad-Dahna deserts) contained the lowest number of high conservation priority species and the lowest summed conservation priority scores in the Kingdom. Not surprisingly, these hyper-arid regions also contain the lowest number of breeding species in Saudi Arabia (Boland and Alsuhaibany 2020). These results suggest that in order to protect Saudi Arabia's highest conservation priority bird species, conservation agencies and landowners should increase protection efforts in the southwestern regions of the Kingdom, particularly the highlands.

\section{Threats to Saudi Arabia's high conservation priority species}

Because of this ranking system we are now able to assess the threatening processes impacting the highest conservation priority species in Saudi Arabia (Table 3). Although very little conservation research has been devoted to the birds of Saudi Arabia, our preliminary analysis indicates that a wide variety of threats are impacting Saudi Arabia's highest conservation priority birds, particularly hunting, alpine habitat loss / degradation, Allee effects on small populations, secondary poisoning, and persecution, along with numerous other impacts (Table 3). Conservation programs targeted at reducing the impacts of hunting, and protecting and restoring habitat in the Asir Mountains are clearly urgent priorities. We recommend developing species-specific recovery plans for the 20 highest conservation priority bird species in Saudi Arabia.

\section{CONCLUSION}

We developed a species conservation prioritization system to rank the birds of Saudi Arabia that is transparent and can be readily applied to other vertebrate taxa and in other regions, including those with limited baseline data about species' national distributions and abundances. The methodology should be applied to all vertebrate taxa and countries within the Arabian Peninsula as a means of informing regional conservation efforts. As the largest country in the Arabian Peninsula, the efforts of Saudi Arabia are likely to be pivotal to the conservation outcomes for all birds listed as high conservation priority species. 
Table 3. Key threatening processes for the 20 highest conservation priority bird species in Saudi Arabia.

\begin{tabular}{|c|c|c|c|c|}
\hline Rank & Species & Score & Key threats within Saudi Arabia & References \\
\hline 1 & $\begin{array}{l}\text { Asir Magpie } \\
\text { Pica asirensis }\end{array}$ & 87.8 & Alpine habitat loss / degradation, Allee effects & Babbington 2016, Boland and Burwell 2020 \\
\hline 2 & $\begin{array}{l}\text { Basra Reed-Warbler } \\
\text { Acrocephalus griseldis }\end{array}$ & 62.0 & Hunting & Brochet et al. 2019 \\
\hline 3 & $\begin{array}{l}\text { Socotra Cormorant } \\
\text { Phalacrocorax nigrogularis }\end{array}$ & 61.6 & Island habitat degradation, introduced predators & Whelan et al. 2018, Khan et al. 2019 \\
\hline 4 & $\begin{array}{l}\text { Arabian Woodpecker } \\
\text { Dendropicos dorae }\end{array}$ & 60.8 & Alpine habitat loss / degradation & Jennings 2010, Boland and Alsuhaibany 2020 \\
\hline 5 & $\begin{array}{l}\text { Yemen Warbler } \\
\text { Sylvia buryi }\end{array}$ & 60.2 & Alpine habitat loss / degradation & Jennings 2010, Boland and Alsuhaibany 2020 \\
\hline 6 & $\begin{array}{l}\text { Asian Houbara } \\
\text { Chlamydotis macqueenii }\end{array}$ & 59.8 & Hunting & Tourenq et al. 2005, Brochet et al. 2019, Nabi et al. 2019 \\
\hline 7 & $\begin{array}{l}\text { Yemen Thrush } \\
\text { Turdus menachensis }\end{array}$ & 59.0 & Alpine habitat loss / degradation & Jennings 2010, Boland and Alsuhaibany 2020 \\
\hline 8 & $\begin{array}{l}\text { Arabian Grosbeak } \\
\text { Rhynchostruthus percivali }\end{array}$ & 58.8 & Alpine habitat loss / degradation & Jennings 2010, Boland and Alsuhaibany 2020 \\
\hline 9 & $\begin{array}{l}\text { Arabian Bustard } \\
\text { Ardeotis arabs }\end{array}$ & 58.3 & Allee effects, hunting & Brochet et al. 2019, Boland and Alsuhaibany 2020 \\
\hline 10 & $\begin{array}{l}\text { Northern Bald Ibis } \\
\text { Geronticus eremita }\end{array}$ & 52.3 & Allee effects, hunting & Lindsell et al. 2009, Serra et al. 2011, Brochet et al. 2019 \\
\hline 11 & $\begin{array}{l}\text { Bearded Vulture } \\
\text { Gypaetus barbatus }\end{array}$ & 51.7 & Secondary poisoning, persecution & Ogada et al. 2012, Plaza et al. 2019 \\
\hline 12 & $\begin{array}{l}\text { Sooty Falcon } \\
\text { Falco concolor }\end{array}$ & 51.5 & Trapping, substandard falconry practices, hunting & $\begin{array}{l}\text { Gaucher et al. 1995, McGrady et al. 2016, Brochet et al. } \\
2019\end{array}$ \\
\hline 13 & $\begin{array}{l}\text { Crab Plover } \\
\text { Dromas ardeola }\end{array}$ & 51.0 & $\begin{array}{l}\text { Human disturbance, egg collection, introduced } \\
\text { predators }\end{array}$ & Almalki et al. 2014 \\
\hline 14 & $\begin{array}{l}\text { Common Ostrich } \\
\text { Struthio camelus }\end{array}$ & 50.5 & Drought, hunting, Allee effects & Islam et al. 2008 \\
\hline 15 & $\begin{array}{l}\text { Lappet-faced Vulture } \\
\text { Torgos tracheliotos }\end{array}$ & 49.7 & $\begin{array}{l}\text { Secondary poisoning, persecution, nest } \\
\text { disturbance }\end{array}$ & Shobrak 2003, Ogada et al. 2012, Plaza et al. 2019 \\
\hline 16 & $\begin{array}{l}\text { Arabian Waxbill } \\
\text { Estrilda rufibarba }\end{array}$ & 49.6 & Alpine and foothills habitat loss / degradation & Jennings 2010, Boland and Alsuhaibany 2020 \\
\hline 17 & $\begin{array}{l}\text { Tawny Eagle } \\
\text { Aquila rapax }\end{array}$ & 48.7 & Secondary poisoning, hunting, persecution & Brown 1991, Brochet et al. 2019 \\
\hline 18 & $\begin{array}{l}\text { Arabian Lark } \\
\text { Eremalauda eremodites }\end{array}$ & 48.3 & Desert habitat loss / degradation, hunting & Brochet et al. 2019, Boland and Alsuhaibany 2020 \\
\hline 19 & $\begin{array}{l}\text { Cinereous Bunting } \\
\text { Emberiza cineracea }\end{array}$ & 47.5 & Hunting & Brochet et al. 2019 \\
\hline 20 & $\begin{array}{l}\text { Philby's Partridge } \\
\text { Alectoris philbyi }\end{array}$ & 47.3 & Alpine habitat loss / degradation, hunting & Brochet et al. 2019, Boland and Alsuhaibany 2020 \\
\hline
\end{tabular}

Responses to this article can be read online at:

http://www.ace-eco.org/issues/responses.php/1705

\section{Acknowledgments:}

We thank Saudi Aramco for its ongoing commitment to conserving biodiversity and two anonymous reviewers for comments on a previous version of the manuscript.

\section{LITERATURE CITED}

Almalki, M., M. AlRashidi, M. Shobrak, and T. Székely. 2014. Breeding distribution and conservation of the Crab Plover (Dromas ardeola) in Saudi Arabia (Aves: Charadriiformes). Zoology in the Middle East 60(1):6-12. https://doi. org/10.1080/09397140.2014.892300

Avery, M., D. W. Gibbons, R. Porter, T. Tew, G. Tucker, and G. Williams. 1995. Revising the British Red Data List for birds: the biological basis of UK conservation priorities. Ibis 137:S232S239. https://doi.org/10.1111/j.1474-919X.1995.tb08450.x
Babbington, J. 2016. Update on the status and occurrence of Arabian Magpie Pica pica asirensis in Saudi Arabia. Sandgrouse 38(2):146-151.

Barnosky, A. D., N. Matzke, S. Tomiya, G. O. Wogan, B. Swartz, T. B. Quental, C. Marshall, J. L. McGuire, E. L. Lindsey, K. C. Maguire, B. Mersey, E. A. Ferrer. 2011. Has the Earth's sixth mass extinction already arrived? Nature 471:51-57. https://doi. org/10.1038/nature09678

Bennett, J. R., G. Elliott, B. Mellish, L. N. Joseph, A. I. Tulloch, W. J. Probert, M. M. Di Fonzo, J. M. Monks, H. P. Possingham, and R. Maloney. 2014. Balancing phylogenetic diversity and species numbers in conservation prioritization, using a case study of threatened species in New Zealand. Biological Conservation 174:47-54. https://doi.org/10.1016/j.biocon.2014.03.013

Boland, C. R. J., and B. O. Burwell. 2020. Habitat modeling reveals extreme habitat fragmentation in the endangered and declining Asir magpie, Pica asirensis, Saudi Arabia's only endemic bird (Aves: Passeriformes). Zoology in the Middle East. https:// doi.org/10.1080/09397140.2020.1833471

Boland, C. R. J., and A. H. Alsuhaibany. 2020. The birds of Saudi Arabia. Motivate, Dubai, United Arab Emirates. 
Bottrill, M. C., L. N. Joseph, J. Carwardine, M. Bode, C. Cook, E. T. Game, H. Grantham, S. Kark, S. Linke, E. McDonaldMadden, R. L. Pressey, S. Walker, K. A. Wilson, and H. P. Possingham. 2008. Is conservation triage just smart decision making? Trends in Ecology and Evolution 23(12):649-654. https:// doi.org/10.1016/j.tree.2008.07.007

Bowen-Jones, E., and A. Entwistle. 2002. Identifying appropriate flagship species: the importance of culture and local contexts. Oryx 36(2):189-195. https://doi.org/10.1017/S0030605302000261

Brochet, A. L., S. Jbour, R. D. Sheldon, R. Porter, V. R. Jones, W. Al Fazari, O. Al Saghier, S. Alkhuzai, L. A. Al-Obeidi, R. Angwin, K. Ararat, M. Pope, M. Y. Shobrak, M. S. Willson, S. S. Zadegan, and S. H. M. Butchart. 2019. Preliminary assessment of the scope and scale of illegal killing and taking of wild birds in the Arabian Peninsula. Sandgrouse 41:154-175.

Brown, C. J. 1991. Declining Martial Polemaetus bellicosus and Tawny Aquila rapax Eagle populations and causes of mortality on farmlands in central Namibia. Biological Conservation 56 (1):49-62. https://doi.org/10.1016/0006-3207(91)90088-Q

Conservation International. 2020. Critical ecosystem partnership fund. Explore the biodiversity hotspots. Conservation International. [online] URL: https://www.cepf.net/our-work/ biodiversity-hotspots

del Hoyo, J., A. Elliott, J. Sargatal, D. A. Christie, and E. de Juana. 2020. Handbook of the birds of the world alive. Lynx Edicions, Barcelona, Spain.

Ducarme, F., G. M. Luque, and F. Courchamp. 2013. What are "charismatic species" for conservation biologists? BioSciences Master Reviews 10:1-8.

Environmental Systems Research Institute (ESRI). 2018. ArcGIS desktop release 10.6.1. Environmental Systems Research Institute, Redlands, California, USA.

Fischer, A., B. Bednar-Friedl, F. Langers, M. Dobrovodská, N. Geamana, K. Skogen, and M. Dumortier. 2011. Universal criteria for species conservation priorities? Findings from a survey of public views across Europe. Biological Conservation 144 (3):998-1007. https://doi.org/10.1016/j.biocon.2010.12.004

Gaucher, P., J. M. Thiollay, and X. Eichaker. 1995. The Sooty Falcon Falco concolor on the Red Sea coast of Saudi Arabia: distribution, numbers and conservation. Ibis 137(1):29-34. https://doi.org/10.1111/j.1474-919X.1995.tb03216.x

Gauthier, P., M. Debussche, and J. D. Thompson. 2010. Regional priority setting for rare species based on a method combining three criteria. Biological Conservation 143(6):1501-1509. https:// doi.org/10.1016/j.biocon.2010.03.032

Holt, D. W., R. Berkley, C. Deppe, P. L. Enríquez Rocha, J. L. Petersen, J. L. Rangel Salazar, K. P. Segars, K. L. Wood, and J. S. Marks. 2020. Spotted Eagle-Owl (Bubo africanus), version 1.0. In J. del Hoyo, A. Elliott, J. Sargatal, D. A. Christie, and E. de Juana, editors. Birds of the world. Cornell Lab of Ornithology, Cornell, New York, USA. https://doi.org/10.2173/bow. speowl2.01

Isaac, N. J. B., S. T. Turvey, B. Collen, C. Waterman, and J. E. M. Baillie. 2007. Mammals on the EDGE: conservation priorities based on threat and phylogeny. PLoS ONE 2(3):e296. https://doi. org/10.1371/journal.pone.0000296

International Union for Conservation of Nature (IUCN). 2020. The IUCN Red List of Threatened Species. Version 2020-1. IUCN Global Species Programme Red List Unit, Cambridge, UK. [online] URL: https://www.iucnredlist.org

Islam, M. Z., A. Singh, M. P. Basheer, J. Judas, and A. Boug. 2013. Differences in space use and habitat selection between captive-bred and wild-born houbara bustards in Saudi Arabia: results from a long-term reintroduction program. Journal of Zoology 289(4):251-261. https://doi.org/10.1111/jzo.12002

Islam, Z. M., K. Ismail, and A. Boug. 2008. Re-introduction of the Red-necked Ostrich, Struthio camelus camelus, in Mahazat as-Sayd Protected Area in central Saudi Arabia. Zoology in the Middle East 44(1):31-40. https://doi.org/10.1080/09397140.2008.10638286

James, A., K. J. Gaston, and A. Balmford. 2001. Can we afford to conserve biodiversity? BioScience 51(1):43-52. https://doi. org/10.1641/0006-3568(2001)051[0043:cwatcb]2.0.co;2

Jennings, M. C. 2010. Atlas of the Breeding Birds of Arabia. Fauna of Arabia 25:1-751.

Jepson, P., and M. Barua. 2015. A theory of flagship species action. Conservation and Society 13(1):95-104. https://doi. org/10.4103/0972-4923.161228

Jetz, W., G. H. Thomas, J. B. Joy, D. W. Redding, K. Hartmann, and A. O. Mooers. 2014. Global distribution and conservation of evolutionary distinctiveness in birds. Current Biology 24 (9):919-930. https://doi.org/10.1016/j.cub.2014.03.011

Joseph, L. N., R. F. Maloney, and H. P. Possingham. 2009. Optimal allocation of resources among threatened species: a project prioritization protocol. Conservation Biology 23 (2):328-338. https://doi.org/10.1111/j.1523-1739.2008.01124.x

Khan, S. B., S. Javed, S. Ahmed, E. A. Al Hammadi, A. A. Al Hammadi, and S. Al Dhaheri. 2019. Does a recent surge in Socotra Cormorant Phalacrocorax nigrogularis nesting population and establishment of new breeding colonies ensure long term conservation? Pragmatic assessment of recent augmentation in Abu Dhabi Emirate, UAE. Bird Conservation International 29(3):361-369. https://doi.org/10.1017/S0959270918000242

Kukkala, A., L. Maiorano, W. Thuiller, and A. Arponen. 2019. Identifying national responsibility species based on spatial conservation prioritization. Biological Conservation 236:411-419. https://doi.org/10.1016/j.biocon.2019.05.046

Lambeck, R. J. 1997. Focal species: a multi-species umbrella for nature conservation. Conservation Biology 11(4):849-856. https:// doi.org/10.1046/j.1523-1739.1997.96319.x

Le Berre, M., V. Noble, M. Pires, F. Médail, and K. Diadema. 2019. How to hierarchize species to determine priorities for conservation action? A critical analysis. Biodiversity and Conservation 28:3051-3071. https://doi.org/10.1007/s10531-019-01820$\mathrm{w}$

Lindenmayer, D. B., C. R. Margules, and D. B. Botkin. 2000. Indicators of biodiversity for ecologically sustainable forest management. Conservation Biology 14(4):941-950. https://doi. org/10.1046/j.1523-1739.2000.98533.x 
Lindsell, J. A., G. Serra, L. Peške. M. S. Abdullah, G. al Qaim, A. Kanani, and M. Wondafrash. 2009. Satellite tracking reveals the migration route and wintering area of the Middle East population of critically endangered northern bald ibis Geronticus eremita. Oryx 43(3):329-335. https://doi.org/10.1017/S0030605309001963

Mace, G. M., and E. J. Hudson. 1999. Attitudes toward sustainability and extinction. Conservation Biology 13 (2):242-246.

Mace, G. M., H. P. Possingham, and N. Leader-Williams. 2007. Prioritizing choices in conservation. Pages 17-34 in D. W. Macdonald and K. J. Service, editors. Key topics in conservation biology. Blackwell, Hoboken, New Jersey, USA.

McCarthy, M. A., C. J. Thompson, and S. T. Garnett. 2008. Optimal investment in conservation of species. Journal of Applied Ecology 45(5):1428-1435. https://doi.org/10.1111/j.1365-2664.2008.01521. $\mathrm{x}$

McGowan, J., L. J. Beaumont, R. J. Smith, A. L. Chauvenet, R. Harcourt, S. C. Atkinson, J. C. Mittermeier, M. EsperonRodriguez, J. B. Baumgartner, A. Beattie, et al. 2020. Conservation prioritization can resolve the flagship species conundrum. Nature Communications 11:994. https://doi. org/10.1038/s41467-020-14554-z

McGrady, M. J., W. A. Al Fazari, M. H. Al Jahdhami, J. E. Hines, and M. K. Oli. 2016. Survival of Sooty Falcons (Falco concolor) breeding in Oman. Journal of Ornithology 157:427-437. https:// doi.org/10.1007/s10336-015-1302-6

Meyer, C., H. Kreft, R. Guralnick, and W. Jetz. 2015. Global priorities for an effective information basis of biodiversity distributions. Nature Communications 6:8221. https://doi. org/10.1038/ncomms9221

Nabi, G., R. Ullah, S. Khan, Nawsherwan, M. Amin, and N. Rauf. 2019. The Asian Houbara Bustard (Chlamydotis macqueenii): on an accelerating path to extinction? Biodiversity and Conservation 28:1301-1302. https://doi.org/10.1007/ s10531-019-01727-6

National Commission for Wildlife Conservation and Development (NCWCD). 2003. First Saudi Arabian national report on the Convention on Biological Diversity. NCWCD, Riyadh, Kingdom of Saudi Arabia.

Ogada, D. L., F. Keesing, and M. Z. Virani. 2012. Dropping dead: causes and consequences of vulture population declines worldwide. Annals of the New York Academy of Sciences 1249 (1):57-71. https://doi.org/10.1111/j.1749-6632.2011.06293.x

Pimm, S. L., G. J. Russell, J. L. Gittleman, and T. M. Brooks. 1995. The future of biodiversity. Science 269(5222):347-350. https://doi.org/10.1126/science.269.5222.347

Plaza, P. I., E. Martínez-López, and S. A. Lambertucci. 2019. The perfect threat: pesticides and vultures. Science of the Total Environment 687:1207-1218. https://doi.org/10.1016/j.

scitotenv.2019.06.160

Redding, D. W., K. Hartmann, A. Mimoto, D. Bokal, M. DeVos, and A. Ø. Mooers. 2008. Evolutionarily distinctive species often capture more phylogenetic diversity than expected. Journal of Theoretical Biology 251(4):606-615. https://doi.org/10.1016/j. jtbi.2007.12.006
Roberge, J.-M., G. Mikusiński, and S. Svensson. 2008. The Whitebacked Woodpecker: umbrella species for forest conservation planning? Biodiversity and Conservation 17(10):2479-2494. https://doi.org/10.1007/s10531-008-9394-4

Rodríguez, J. P., F. Rojas-Suárez, and C. J. Sharpe. 2004. Setting priorities for the conservation of Venezuela's threatened birds. Oryx 38(4):373-382. https://doi.org/10.1017/S0030605304000730

Schmeller, D. S., B. Gruber, E. Budrys, E. Framsted, S. Lengyel, and K. Henle. 2008. National responsibilities in European species conservation: a methodological review. Conservation Biology 22 (3):593-601. https://doi.org/10.1111/j.1523-1739.2008.00961.x

Schmeller, D. S., D. Evans, Y.-P. Lin, and K. Henle. 2014. The national responsibility approach to setting conservation priorities-recommendations for its use. Journal for Nature Conservation 22(4):349-357. https://doi.org/10.1016/j.jnc.2014.03.002

Schnittler, M., and K.-F. Günther. 1999. Central European vascular plants requiring priority conservation measures-an analysis from national Red Lists and distribution maps. Biodiversity and Conservation 8:891-925. https://doi.org/10.1023/ a:1008828704456

Seoane, J., L. M. Carrascal, and D. Palomino. 2011. Assessing the ecological basis of conservation priority lists for bird species in an island scenario. Journal for Nature Conservation 19 (2):103-115. https://doi.org/10.1016/j.jnc.2010.07.002

Serra, G., C. Bruschini, J. A. Lindsell, L. Peske, A. and Kanani. 2011. Breeding range of the last eastern colony of Critically Endangered Northern Bald Ibis Geronticus eremita in the Syrian steppe: a threatened area. Bird Conservation International 21 (3):284-295. https://doi.org/10.1017/S095927091000064X

Shobrak, M. 2003. Vultures in Saudi Arabia. Vulture News 48:20-23.

Simberloff, D. 1998. Flagships, umbrellas, and keystones: is single-species management passé in the landscape era? Biological Conservation 83(3):247-257. https://doi.org/10.1016/s0006-3207 (97)00081-5

Small, E. 2011. The new Noah's Ark: beautiful and useful species only. Part 1. Biodiversity conservation issues and priorities. Biodiversity 12(4):232-247. https://doi.org/10.1080/14888386.2011.642663

Symes, A., J. Taylor, D. Mallon, R. Porter, C. Simms, and K. Budd. 2015. The conservation status and distribution of the breeding birds of the Arabian Peninsula. IUCN, Cambridge, UK and Gland, Switzerland, and Environment and Protected Areas Authority, Sharjah, UAE. https://doi.org/10.2305/IUCN.CH.2015.MRA.5. en

Tourenq, C., O. Combreau. M. Lawrence, S. B. Pole, A. Spalton, G. Xinji, M. Al Baidani and F. Launay. 2005. Alarming Houbara Bustard population trends in Asia. Biological Conservation 121 (1):1-8. https://doi.org/10.1016/j.biocon.2004.03.031

Vane-Wright, R. I., C. J. Humphries, and P. H. Williams. 1991. What to protect? Systematics and the agony of choice. Biological Conservation 55(3):235-254. https://doi.org/10.1016/0006-3207 (91)90030-D

Vieira da Silva, C., L. Madureira, J. L. Costa, and J. L. Santos. 2016. Using public preferences to weight species prioritization 
criteria in conservation decision making: a case study to explore potential strengths and weaknesses. Society and Natural Resources 29(12):1517-1532. https://doi.org/10.1080/08941920.2016.1171937

Wells, J. V., B. Robertson, K. V. Rosenberg, and D. W. Mehlman. 2010. Global versus local conservation focus of U.S. state agency endangered bird species lists. PLOS ONE 5(1):e8608. https://doi. org/10.1371/journal.pone.0008608

Whelan, R., C. Clarke, N. Almansoori, A. Jaradat, N. S. Al Qadi, and S. B. Muzaffar. 2018. Demographic consequences of native fox predation on Socotra Cormorants on Siniya Island, United Arab Emirates. Wildlife Biology 2018(1). https://doi.org/10.2981/ wlb.00450

Wilson, K. A., J. Carwardine, and H. P. Possingham. 2009. Setting conservation priorities. Annals of the New York Academy of Sciences 1162(1):237-264. https://doi.org/10.1111/j.1749-6632.2009.04149. $\mathrm{x}$

Wilson, K. A., M. F. McBride, M. Bode, and H. P. Possingham. 2006. Prioritizing global conservation efforts. Nature 440:337-340. https://doi.org/10.1038/nature04366

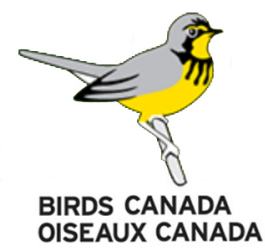

